Running head: The mechanisms of prediction updating

The mechanisms of prediction updating that impact the processing of upcoming words an event-related potential study on sentence comprehension

Jakub M. Szewczyk; Zofia Wodniecka

Institute of Psychology, Jagiellonian University, Kraków, Poland

Manuscript published in

Journal of Experimental Psychology: Learning, Memory, and Cognition

https://doi.org/10.1037/xlm0000835

(version accepted for publication)

Author note

All materials, data (including raw EEG) and analysis scripts are available on OSF:

https://osf.io/c6hxt.

Correspondence address:

Jakub M. Szewczyk, Department of Psychology, University of Illinois at Urbana-Champaign, 603 East Daniel St., Champaign, IL 61820. E-mail: jakub.szewczyk@ gmail.com 


\begin{abstract}
One of the most compelling pieces of evidence for the presence of predictions in language comprehension comes from event-related potential (ERP) studies which show that encountering an adjective whose gender marking is inconsistent with that of a highly expectable noun leads to an effect at the adjective. Until now the mechanism underlying this effect has been unknown. The present study tests a novel hypothesis whereby the effect at adjectives reflects prediction updating, which in turn impacts the N400 generated by the upcoming noun. In two experiments, native speakers of Polish read short stories that made several nouns likely at the position of a direct object in the story's final sentence. The noun was preceded by a gender-marked adjective which was shown in either of the two grammatical forms: a) informative about the following noun (adjectival gender marking constrained which nouns can follow), or b) less informative (the grammatical marking did not disambiguate the gender of the following noun). Importantly, the same stories and critical words were used in both conditions, which was possible thanks to exploiting syncretisms in Polish adjectival inflection. The results provide support for one or more mechanisms of prediction updating at the adjective that impact the amplitude of the N400

elicited by the following noun. Together, these results show that when only morphosyntactic cues are available, they are instantly used to update predictions about upcoming words.
\end{abstract}

Keywords: sentence comprehension, predictions, prediction updating, delayed N400, surprisal 
The mechanisms of prediction updating that impact the processing of upcoming words - an event-related potential study on sentence comprehension

It has been widely demonstrated that predictable words are easier to process: people are faster to read, name, or make lexical decisions on contextually supported words (e.g. Duffy, Henderson, \& Morris, 1989; Ehrlich \& Rayner, 1981; Kleiman, 1980; McClelland \& O’Regan, 1981; Rayner \& Well, 1996; Schwanenflugel \& LaCount, 1988; Schwanenflugel \& Shoben, 1985; Schwanenflugel \& White, 1991); such words also elicit a reduced N400 ERP component ${ }^{1}$ (see Kutas, DeLong, \& Smith, 2011 for a review) and evoke less metabolic activity in the left temporal cortex (Carter, Foster, Muncy, \& Luke, 2019; Frank \& Willems, 2017; Henderson, Choi, Lowder, \& Ferreira, 2016; (Schuster, Hawelka, Hutzler, Kronbichler, \& Richlan, 2016; Willems, Frank, Nijhof, Hagoort, \& van den Bosch, 2016). One of the challenges in the early days was clearly demonstrating that readers or listeners predict ${ }^{2}$ upcoming parts of a text or message. Researchers were initially reluctant to acknowledge that the effects of predictability might be a result of prediction and instead suggested that the predictability effects can be explained as a downstream consequence of the processing difficulty of the word (Brown \& Hagoort, 1993; Van Berkum, Hagoort, \& Brown, 1999). For example, it has been argued that less predictable words may simply be more difficult to integrate with their semantic context.

These interpretational controversies were circumvented by demonstrating the predictive effects that occur even before the predictable word is encountered. This was made possible with ERPs and eye-tracking techniques that allow online word-by-word tracing of language comprehension while participants listen to or read short stories (Altmann \& Kamide, 1999; Wicha, Moreno, \& Kutas, 2004). Within ERPs, a paradigm that particularly helped in showing predictions was the "prenominal words" paradigm. In the two experiments reported in the current paper, we used this paradigm to go beyond mere demonstrations of predictions. We aimed to understand the mechanisms that underlie prediction-related effects.

\section{The "prenominal words" paradigm}

The "prenominal words" paradigm was first demonstrated by Wicha et al. (2004) and van Berkum et al. (2005) and has subsequently been used in many other studies (e.g. DeLong, Groppe, Urbach, \& Kutas, 2012; DeLong, Urbach, \& Kutas, 2005; Foucart, Martin, Moreno, \& Costa, 2014; Martin et al., 2013; Otten, Nieuwland, \& Van Berkum, 2007; Otten \& Van Berkum, 2008, 2009; but see: Nieuwland et al., 2018; Kochari \& Flecken, 2018 for failed replications). For example, Szewczyk and Schriefers (2013) asked participants to read short stories in Polish, such as the following:

\footnotetext{
${ }^{1}$ The amplitude of the N400 component is taken to reflect the amount of processing required to access the meaning of the stimulus at hand in long-term memory; the larger the amount of processing required, the more negative the component's amplitude (Kutas \& Federmeier, 2011). Accordingly, words whose meaning is presumably already preactivated by the preceding context elicit an N400 component with a reduced amplitude.

2 In this paper we use the terms 'prediction' and 'preactivation' interchangeably, both of them referring to the situation in which the activation of a word in LTM is increased before the word is encountered in the discourse.
} 
(1a) My mother decided that we should have a 'spring clean' in our house. She cleaned the living room and the kitchen, and my father's job was to clean the first floor. My job was to clean the entire ${ }_{M A S C-I A}$ attic $_{M A S C-I A}$, which had not been used for ages.

[... cały strych ... $]$

The critical noun occurred in a direct object in the final sentence of the story and was preceded by an adjective that agreed in gender with the noun (the noun is masculine inanimate, thus the adjective must carry the $-y$ suffix). In half the items, the direct object noun was replaced by another noun that was unpredictable and semantically incongruent given the context. Moreover, the incongruent noun belonged to a gender class (masculine animate) that was not carried by any of the predictable nouns:

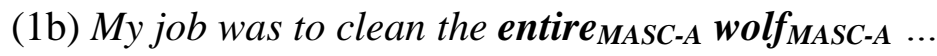

$$
\text { [... całego wilka ... ] }
$$

If participants had predicted the target noun(s), at the preceding adjective they would have realized that the prediction would be violated because the adjective had a suffix (-ego) that mismatched the gender of the predicted noun(s). ERPs time-locked to the critical adjectives (caty ( całego) revealed that the prediction-mismatching adjectives lead to an N400 effect compared to the prediction-matching adjectives. Thus, this ERP effect indicates that participants must have known the gender of the noun; moreover, because the effect occurred before the noun was encountered, this indicates that participants must have predicted the noun. Most other studies on predictions also found similar negativities for the comparison between prediction-matching and prediction-mismatching words preceding nouns (DeLong et al., 2012, 2005; Foucart et al., 2014; Martin et al., 2013; Otten et al., 2007; Otten \& Van Berkum, 2008, 2009; Szewczyk \& Schriefers, 2013; Wicha, Bates, Moreno, \& Kutas, 2003; Wicha, Moreno, \& Kutas, 2003).

In this paper, we will focus on the effect evoked by prediction-mismatching words and ask which alternative mechanism it is that the ERP effect reflects. One possibility is that it simply reflects error detection: the brain notices the mismatch between its expectations and the actual input (see e.g. Dikker \& Pylkkänen, 2013, p. 56). The other possibility, formulated as the prediction updating hypothesis (Szewczyk, 2016; Szewczyk \& Schriefers, 2018), proposed that not only does the language comprehension system notice the discrepancy between its predictions and the form of the adjective, but it also uses this information to update the predictions about the noun. The central goal of this paper is to adjudicate between the two possible interpretations of the N400 effect on the adjective: 1) just noticing the mismatch or 2) noticing the mismatch and updating the predictions. To this aim, we will test whether the effect at the adjective has causal consequences for the processing of the noun.

The prediction updating hypothesis (Szewczyk, 2016; Szewczyk \& Schriefers, 2018) posits that by the time the adjective is encountered, some predictions about the noun are already 
formulated (in the mini-story given in examples $1 \mathrm{a}$ and $1 \mathrm{~b}$, a set of words like attic, basement, bathroom, etc.). "Prediction" here refers to pre-activation - the notion that the semantic or conceptual representations of the possible upcoming nouns are activated in long-term memory before the actual noun appears. However, it does not imply that these nouns are already part of the context representation (pre-integration) or that nouns' orthographic representations are also preactivated (prediction of form). This type of prediction (pre-activation) can be probed by comparing the amplitude of the N400 to a noun which has been activated to a larger or smaller extent due to an experimental manipulation (Kutas \& Federmeier, 2011; Lau \& Namyst, 2019; Szewczyk \& Schriefers, 2018).

We proposed that the level of activation of the conceptual representation of each noun is proportional to its predictability. However, these levels of activation can be changed (i.e. the predictions can be updated) when an informative cue is encountered before the noun: in the example above, this is the suffix of the prenominal adjective. Upon encountering the adjective, comprehenders match the gender indicated by the adjectival suffix with the genders of the preactivated nouns (attic, basement, bathroom, etc.). If some but not all of the preactivated nouns match the adjectival suffix (such as in 1a), the parser uses this information to update the predictions - to shift the distribution of activation to the matching nouns at the expense of the nouns that do not match the adjectival suffix. In a more extreme case in which none of the preactivated nouns matches the adjectival suffix (such as in 1b), the parser searches for new nouns that would match the gender of the adjective.

Within the prediction updating hypothesis, we proposed that the process of activating new nouns that match the adjective is reflected at the adjective by the N400 component (Szewczyk, 2016; Szewczyk \& Schriefers, 2018), which reflects the neurocognitive mechanism that is responsible for accessing meaning representations in long-term memory (Kutas \& Federmeier, 2011). The prediction updating hypothesis makes two critical predictions: 1) the amplitude of the N400 to the adjective is proportional to the amount of updating; 2) the update increases the level of activation of the subsequent noun (indexed by the N400 component at the noun) proportionally to the amount of updating.

We tested both these predictions in the reported experiments. We used the experimental design to manipulate the amount of updating at the adjective within-item, using the same or almost the same contexts, adjectives, and nouns; this enabled us to reveal a causal relationship between the amount of updating at the adjective and the activation of the noun. To this end, we exploited unique properties of the Polish language and the fact that some grammatical suffixes are more informative than others.

\section{Present study - manipulation of adjectives' informativity}

In Polish, adjectives (and nouns) are inflected with respect to number, case, and gender. Polish has two number classes (singular and plural), seven grammatical cases (two of which will be relevant here: the genitive and the accusative) and five grammatical genders. Each combination of number, case, and gender is associated with a specific adjectival suffix, as shown 
in Table 1. An important property of the Polish inflectional system is that adjectival suffixes show several syncretisms - situations in which the same suffix is associated with more than one cell in the inflectional paradigm. For example, the suffix $-i$ is uniquely associated with masculine inanimate singular adjectives in the accusative case, whereas the suffix -ego is much less unique and denotes several genders in both levels of the number feature (see Table 1). Thus, adjectives ending with - ego are much less informative about the grammatical gender of the head noun than adjectives ending with $-i$.

Table 1

Suffixes used in the inflection of adjectives in Polish, limited to the genitive and accusative cases.

\begin{tabular}{|c|c|c|c|c|}
\hline \multirow[b]{2}{*}{ Gender } & \multicolumn{2}{|c|}{ Genitive } & \multicolumn{2}{|c|}{ Accusative } \\
\hline & SG & PL & SG & $\mathrm{PL}$ \\
\hline MASC-P & -ego & -ich & -ego & $-\mathrm{ich}$ \\
\hline MASC-A & -ego & -ich & -ego & $-e$ \\
\hline MASC-IA & -ego & -ich & $-i / y$ & $-e$ \\
\hline FEM & $-e j$ & -ich & $-a ̨$ & $-e$ \\
\hline NEU & -ego & -ich & $-e$ & $-e$ \\
\hline
\end{tabular}

Note: Abbreviations: MASC-P = masculine personal, MASC-A = masculine animate, MASC-IA $=$ masculine inanimate, $\mathrm{FEM}=$ feminine, $\mathrm{NEU}=$ neuter, $\mathrm{SG}=$ singular, $\mathrm{PL}=$ plural .

\section{Congruent items}

In this study, participants were presented with short stories such as in (2a).

2a) The teachers in our school had been complaining about the bad behavior of children during breaks between classes. The headmaster gathered all the children and delivered a

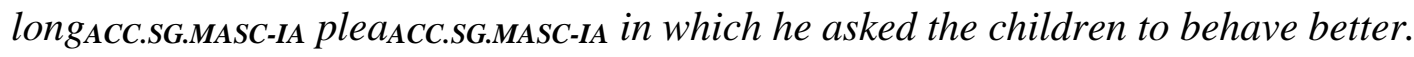
[... długi apel ... ]

Prior to the study, the stories were submitted to a cloze test; it showed that in the above story many nouns are likely as the head of the direct object: speech, rebuke, reprimand, plea, address, sermon, etc. (see Figure 1). In the actual experiment, the story was followed by the noun plea, $\mathrm{p}($ plea $)=.16$. When the noun is preceded by an adjective (long in the actual experiment), participants might be given an additional cue about the gender of the upcoming noun. Plea carries the masculine-inanimate gender, it is singular, and it occurs in the accusative context (direct objects of non-negated verbs are expressed in the accusative case in Polish), so the adjective should carry the suffix $-i$. Because $-i$ is uniquely associated with the masculineinanimate gender, and plea is the only likely noun that carries this gender, the adjectival suffix is highly informative about the upcoming noun: out of all possible (and presumably preactivated at this point) nouns, plea is the only noun that can follow the adjective. Thanks to this, if comprehenders use the information from the adjectival suffix to update their predictions about 
the noun, they should increase the activation of plea to the maximum $(\mathrm{p}($ plea $-i, \mathrm{ACC})=1)$. We refer to this as the more informative variant of this item.

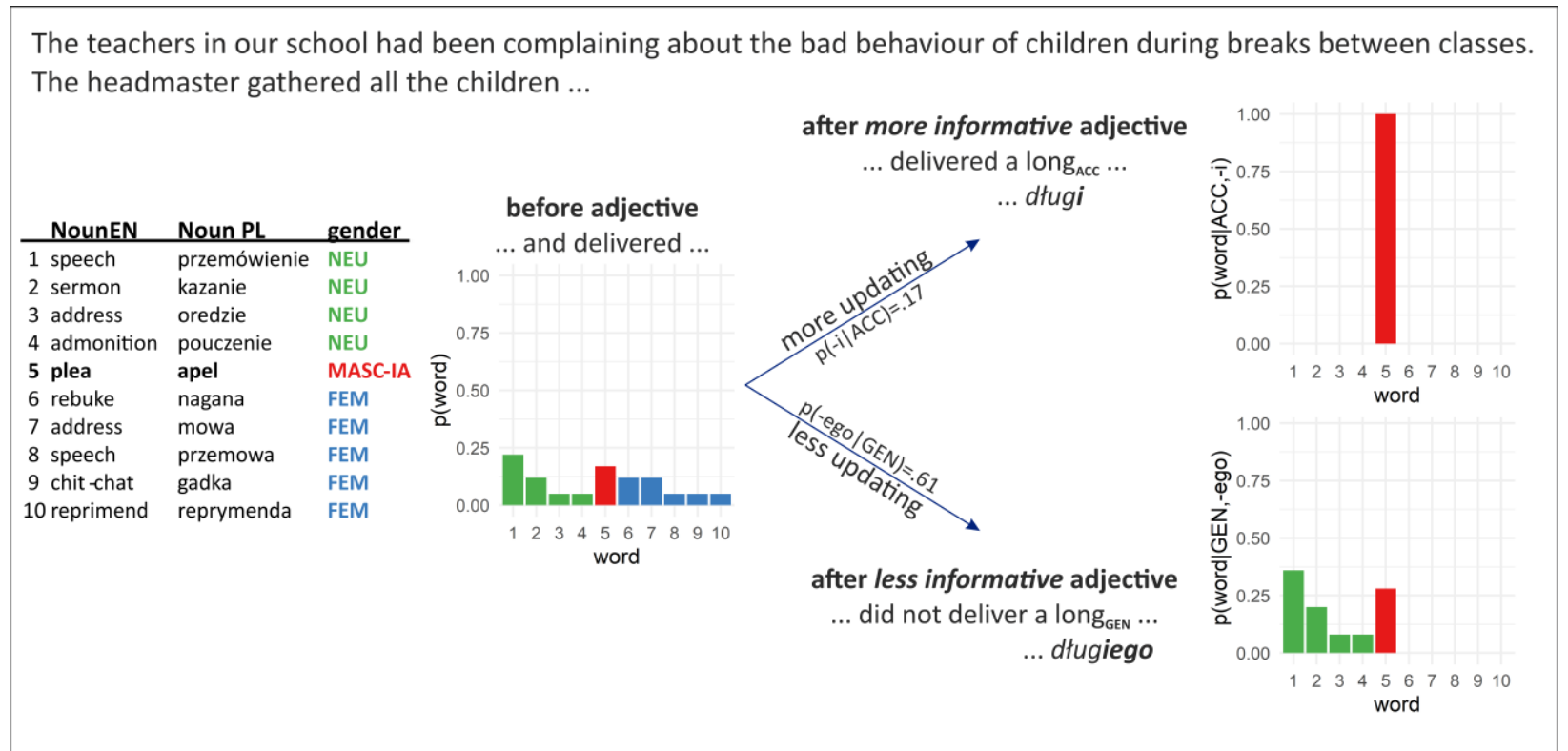

Figure 1. Hypothesized prediction updating in a congruent item. All noun cloze test responses are listed on the left. The numbers presented next to the nouns correspond to the legend on the $\mathrm{x}$ axis in the bar charts. The bar chart on the left shows original cloze values; the charts on the right show hypothesized probabilities of the nouns after being updated by adjectives in the less and more informative conditions.

We can also construct a less informative variant of this story. As already indicated, according to Polish syntax direct objects that follow non-negated verbs are in the accusative case. However, when they follow negated verbs, they are in the genitive case. Thus, by introducing a slight alteration to the story:

2b) ... the headmaster gathered all the children, but surprisingly he did not deliver a longGEN.SG.MASC-IA pleaGEN.SG.MASC-IA ...

$$
\text { [ ... długiego apelu ... ] }
$$

we create a context which requires the direct object to be expressed in the genitive case. Here, the story is also continued by a long plea, but now the adjective has to carry the -ego suffix. As Table 1 shows, this new suffix is more ambiguous: it encodes several grammatical genders and matches several likely nouns. In consequence, the effective cloze probability of the word plea is higher than if there was no adjective but is not as high as in the more informative variant:

$\mathrm{p}($ plea $\mid$-ego, $\mathrm{ACC})=.27$ (see Figure 1$)$.

Thus, depending on whether the verb is negated or not, the conditional probability of the noun plea after seeing the adjective is either 1 (accusative case, non-negated verb, example 2a) or .28 (genitive case, negated verb, example $2 \mathrm{~b}$ ). These two variants of the item correspond to 
the more and the less informative conditions, respectively. Comparing these two variants allowed us to determine whether the adjectival suffix is used to update predictions about the upcoming noun. Critically, the same adjective and noun were used in both variants and they differed only in their grammatical form.

\section{Incongruent items}

In addition to congruent items, in one-third of the items the direct object noun was incongruent:

3a) Some criminals kidnapped the daughter of a well-known businessman and they demanded that she contact her family. The kidnapped girl made a desparate ACC.SG.MASC-A skunkACC.SG.MASC-A begging her family to pay the ransom. [... zdesperowanego skunksa ... ]

The direct object noun skunk is incongruent with the story and, importantly, it does not carry the gender of any of the nouns that are likely in this context (see Figure 2). As previously, the noun is preceded by an adjective. In the above sentence, the adjective is highly informative about the incongruity of the noun because its suffix (-ego) mismatches all the likely nouns, $\mathrm{p}(-$ ego $)=0$.

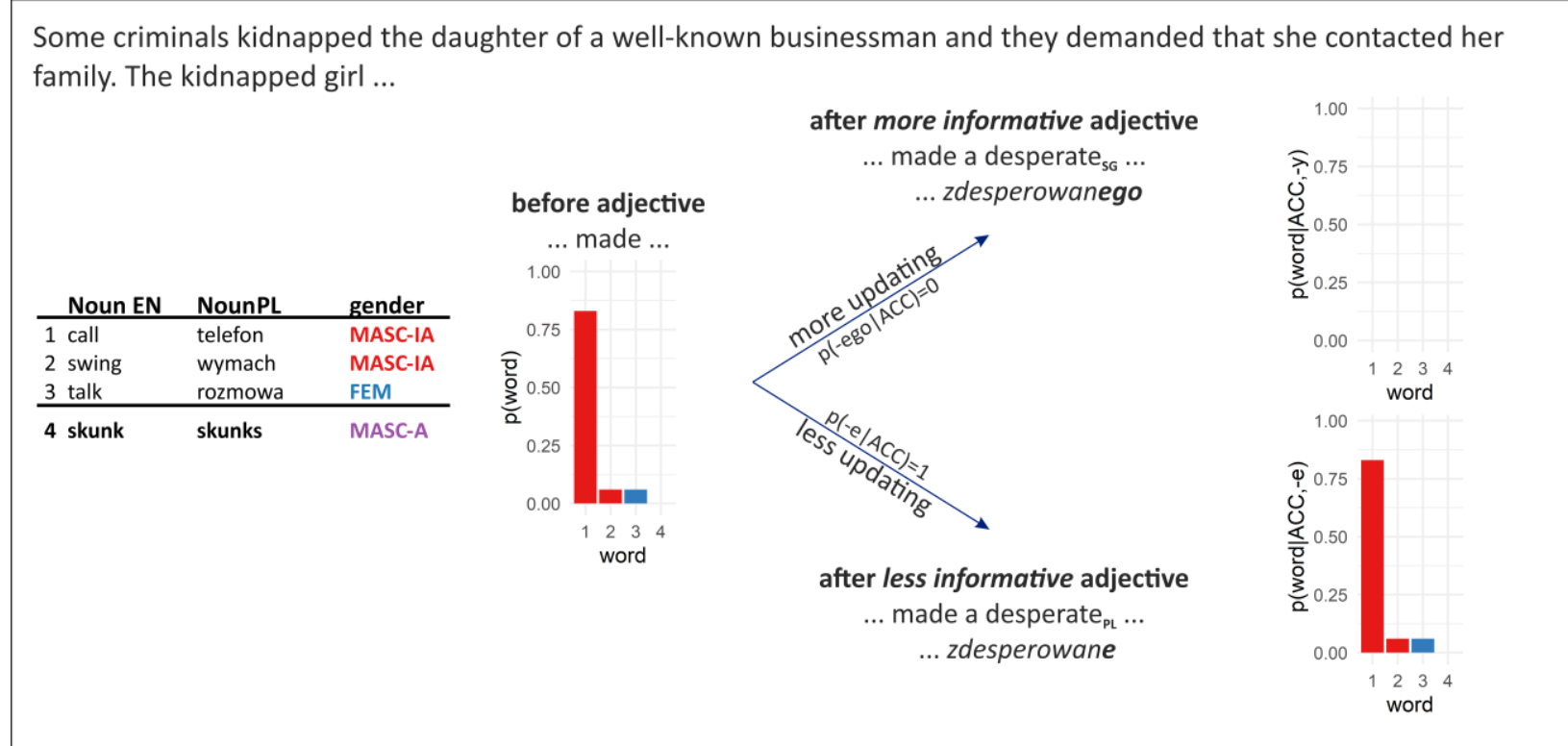

Figure 2. Hypothesized prediction updating in an incongruent item. All noun cloze test responses are listed on the left. Note that skunk is not among them. After the more informative adjective, none of the contextually predictable nouns are possible. Note that English nouns are direct translations from Polish so some of them do not form valid collocations with the English word 'make'. 
As a means to create the less informative variant in this example, we changed the number feature of the direct object (singular to plural) $)^{3}$ :

3b) The kidnapped girl made desparate ACC.PL.MASC-A skunksACC.PL.MASC-A begging ...

$$
\text { [... zdesperowane skunksy ... ] }
$$

The critical adjective carries the suffix $-e$, which, as can be seen in Figure 2, matches all the likely nouns, $\mathrm{p}(-e)=1$. Thus, when the direct object occurs in the plural, the adjective conceals the information that the noun will be incongruent. In either case, the noun remains incongruent and its cloze probability is 0 . The primary reason for the inclusion of the incongruent items was to replicate the N400 effect found on prenominal adjectives in our earlier study (Szewczyk \& Schriefers, 2013). It should be pointed out that the congruent and incongruent items in the present study were essentially identical; the only difference concerned the more informative condition, in which in the incongruent items the adjective did not match any of the predictable nouns, and it was followed by an incongruent noun.

To sum up, in this study we introduced a within-item manipulation of an adjective's informativity (implemented by changing the number feature of the direct object or altering the context to require an accusative vs. genitive direct object). The manipulation was tested separately in the congruent and incongruent items, and each type of item used both ways of manipulating informativity.

\section{ERP predictions}

What kind of ERP effect should reflect prediction updating at the adjective? Past studies testing prediction at informative prenominal words yielded a wide array of ERP effects which varied with respect to time-course, scalp distribution, and sometimes even polarity. At present it is unclear where this variation might come from, but it should be noted that the materials used in these studies differed along many dimensions (e.g., the prenominal word closed vs. open class, visual vs. auditory modality, congruent vs. incongruent items). Moreover, differences in the grammatical features that were employed to test predictions might also contribute to the variation in the obtained ERP effects (e.g. using gender-marked determiners in languages that have two vs. three grammatical genders; see Yan et al. for discussion).

Part of the design of the present study is very similar to our previous study testing semantic predictions (Szewczyk \& Schriefers, 2013) due to the use of the same language, modality, prenominal word type (adjective), and even the largely overlapping set of stories. In the previous study, we compared items in which the noun was congruent or incongruent with the context. The noun was preceded by an adjective whose gender marking indicated whether the predictable congruent noun was likely to appear or not. Thus, the adjectives in the incongruent

${ }^{3}$ In contrast to negating the verb, manipulating the number feature did not require the preceding context to be changed. Manipulations of case and number were done between-item, equally often for congruent and incongruent items. 
condition of that study were like the informative adjectives in incongruent items in the present study. Seen in the light of the prediction updating hypothesis, the adjectives in the congruent condition of the 2013 study required no prediction updating, and thus they were like the less informative adjectives in the current study (congruent and incongruent). The adjectives in the congruent condition of the 2013 study elicited a negativity in the $350-600 \mathrm{~ms}$ time window (in that study we argued that the negativity is a form of N400) relative to adjectives in the incongruent condition. Thus, the analysis of ERPs in the incongruent items of the current study should allow replication of our previous work.

However, our main interest lies in the congruent items. If the prediction updating hypothesis is correct, we can expect to observe an ERP modulation at adjectives in high vs. low informativity conditions in congruent items. This contrast is novel as none of the published studies tested a similar comparison. Our prediction is that an effect will occur at the adjectives, but we remain agnostic about its nature. Because we assume that prediction updating involves changing activation levels of representations of words in long-term memory (LTM), we speculate that the informativity manipulation could result in the $\mathrm{N} 400$ effect on prenominal adjectives, similar to the effect in our 2013 study.

Moving forward to the noun, if the effect at the prenominal adjectives truly reflects prediction updating, it must have causal consequences for the activation of the noun. More specifically, it should reduce the N400 component at the noun because the N400 is known to be an index of preactivation of conceptual representations of the word and of the ease of accessing the word in the current context (Kutas \& Federmeier, 2011; Szewczyk \& Schriefers, 2018). The degree to which the component is reduced should depend on the informativity of the preceding adjectives, with greater reductions following more informative adjectives.

The prediction updating hypothesis assumes that the updating should facilitate the processing of the nouns in the congruent items because the hypothesis assumes that the nouns whose activation is updated should match the adjectival suffix and be congruent with the preceding story. This is certainly the case in congruent items; however, in incongruent items, even if the parser manages to activate new nouns, it is not clear what these nouns would be. The hypothesis assumes that preactivated nouns have to semantically match the context and have gender congruent with the adjectival suffix. However, it is not clear which nouns get preactivated when there are no nouns matching both criteria.

\section{Indexing informativity using graded measures}

In the sections above, we considered the measurement of prediction updating by means of a simple categorical manipulation between more and less informative forms of adjectives. However, the amount of prediction updating can be quantified on a more fine-grained level using continuous indices of information content. Below, we introduce two such indices: surprisal of the suffix and entropy reduction. 


\section{Gender suffix surprisal}

At the adjective, the most basic index is the probability of the adjectival gender suffix, which is computed based on cloze probability tests. It is defined as the sum of cloze probabilities of nouns carrying the gender matching the suffix (see Table 1). The more probable the suffix, the less prediction updating is required because it matches a greater number of probable nouns. For example, the adjective in $3 b$ has a fully likely suffix $(\mathrm{p}(-e \mid \mathrm{ACC})=1$, i.e. the adjective must occur with this suffix) and because it matches nouns of all genders it introduces no new information. As such, this type of adjective leads to no prediction updating ${ }^{4}$ (see Figure 2). Conversely, the adjective in $2 \mathrm{a})$ has a rather unlikely suffix $(\mathrm{p}(-\mathrm{i} \mid \mathrm{ACC})=.17)$ and it can be followed by only one of the nouns that were predictable before the adjective. Thus, the adjective leads to a substantial update of predictions (see Figure 1).

The probability of the gender suffix can also be expressed as surprisal - an index borrowed from Information Theory. Here, it quantifies the improbability or the amount of new information about the noun introduced by the gender information encoded in the adjectival suffix. Because derivation of surprisal involves the logarithm function (surprisal $=-\log (p)$ ), its relation to probability is non-linear: surprisal makes fine distinctions between low and very low probabilities, where probability quickly drops to zero (e.g. the difference between a cloze probability of 0.2 and 0.1 and between 0.02 and 0.01 yields the same difference in surprisal). Several researchers found that word surprisal predicts behavioral and neural indices of language processing difficulty better than probability: reading times (Smith \& Levy, 2013) and the N400 amplitude (Delaney-Busch, Morgan, Lau, \& Kuperberg, 2017; Yan, Kuperberg, \& Jaeger, 2017). As shown by Levy (Levy, 2008) and Yan et al. (2017), surprisal of the adjectival gender suffix also corresponds to Bayesian surprise over the noun semantics incurred by the adjectival suffix (Kullback-Leibler divergence between the probability distribution of likely nouns before and after the adjective), provided that the adjectival suffix is fully determined by the noun. In the case of the present study, suffix surprisal corresponds to the shift between the probability distribution in the set of predictable nouns before and after processing the gender marking on the adjective. Informally, the noun should be less surprising because its gender is already known and other possible nouns mismatching the gender are already excluded by the adjective. Expressing this idea more formally, when a congruent noun is preceded by an adjective with a matching gender suffix, surprisal of the noun should be reduced by the surprisal of the preceding adjective's gender suffix (see Supplementary Materials for a proof). In other words, in congruent items adjectival suffix surprisal should directly reflect the amount of prediction updating. For incongruent items, it is impossible estimate the probability of the incongruent nouns (and in consequence, suffix surprisal) so the analyses using graded measures will be conducted only for congruent items.

\footnotetext{
${ }^{4}$ Note that the mere presence of an adjective informs the parser that the reader has just entered a noun phrase and thus that a head noun is likely to follow. However, in this study an adjective is present in all conditions and thus this syntactic aspect of prediction updating does not differentiate the experimental conditions.
} 


\section{Reduction of predicted nouns' entropy}

Another index that is also borrowed from Information Theory is (single-step) entropy, which in the present study corresponds to the uncertainty about the head noun of the direct object phrase. When many nouns are equally likely, the entropy is high; when only a single noun is likely, the entropy is zero. Noun entropy can be measured at two points: before and after the adjective. When estimated before the adjective, noun entropy corresponds to the entropy of all cloze test completions collected in norming (sentences normed in the cloze tests were truncated before the adjective). When estimated after the adjective, entropy takes into account only a subset of nouns that carry genders matching the adjectival suffix. To account for information brought by the adjectival suffix, we need to compare the noun entropies estimated before and after the adjective. Hale proposed the entropy reduction hypothesis (Hale, 2006; Yun, Chen, Hunter, Whitman, \& Hale, 2015), according to which each reduction in entropy involves cognitive work and thus is associated with increased processing costs. The original hypothesis uses entropy computed over many words ahead (over possible syntactic parses or individual words), and reduction of entropy defined in this way has been demonstrated to predict reading times (Frank, Otten, Galli, \& Vigliocco, 2013; Linzen \& Jaeger, 2015) or sentence-repetition accuracy (Hale, 2006). In this study, we computed reductions in the entropy of a single word only (the direct object noun). Other studies that measured the entropy of the next word brought only mixed results, with some studies finding an effect of entropy reduction (eye-tracking: Lowder, Choi, Ferreira, \& Henderson, 2018; fMRI: Willems, Frank, Nijhof, Hagoort, \& van den Bosch, 2016), while another did not find any (ERPs: Frank et al., 2013).

If the prediction updating hypothesis holds, we anticipate that high adjectival suffix surprisal in congruent items will lead to a larger negativity on the adjective as well as to a smaller N400 component on the noun. It is difficult to foresee the role of entropy-related effects in prediction updating, so this part of the analysis is fully exploratory. This analysis will not be

run for incongruent items because the formula for computing entropy reduction also requires that the probabilities of nouns following informative adjectives are known, and - as indicated previously - these probabilities are difficult to estimate in incongruent items. 


\section{Experiment 1 \\ Methods $^{5}$}

\section{Participants}

Thirty-eight native speakers of Polish were recruited from the subject pool of Jagiellonian University in Kraków, Poland, or via ads on a local job portal. One participant was removed from analyses due to low scores on the story recall test and poor electroencephalogram (EEG) recording quality; this left 37 participants for the final analyses (26 female, mean age: 22.6, range: 19-38). Power calculations conducted before the experiment indicated that at least 32 participants were required to achieve $90 \%$ power to replicate the prediction effect at prenominal adjectives in the study by Szewczyk and Schriefers (2013; the effect size was reduced by $25 \%$ from $d=0.78$ to $d=0.6$ to account for lower number of items in the present study). No participant reported having experienced a neurologic trauma or had any neurologic impairment. Thirty-five participants were right-handed, as assessed by an adapted Polish version of the Edinburgh Handedness Inventory (Oldfield, 1971). Among them, four reported left-handed relatives in their immediate family. The protocol for the experiment was approved by an institutional ethical board.

\section{Materials}

In constructing the materials, we used a database of over 300 stories, many of which were used in our prior experiments (Szewczyk \& Schriefers, 2011, 2013, 2018). All stories were 2-5 sentences long, with the last sentence containing a transitive verb followed by a direct object noun that was the critical word. The critical noun was never sentence-final and all the items were normed for cloze probability at the target noun position. To this end, a separate group of participants was asked to complete stories that were truncated before the direct object noun phrase with the first word that came to mind (minimum 15 ratings per story) ${ }^{6}$.

To select the items for the study, we wrote Python scripts that scoured the database for stories whose pattern of cloze completions (genders and syncretisms in adjectival inflection) allowed informativity to be manipulated using a congruent or incongruent noun (for details of the informativity manipulation, see Introduction). As a result, 97 stories were used in the congruent condition, i.e. the condition in which the target noun was one of the completions proposed in the

\footnotetext{
${ }^{5}$ All materials, raw data and scripts reproducing all analyses and figures are available on https://osf.io/c6hxt/

${ }^{6}$ Original cloze tests were done only for non-negated versions of items (i.e. the critical direct object was shown always in the accusative case). After conducting experiments 1 and 2, for all items in which case manipulation was used we collected additional cloze data separately for the negated and non-negated version of these items (from 29 participants per story in the negated and non-negated versions, each participant saw only one version of each story). Both experiments are analyzed using aggregated data from the original and the new cloze norming. However, we removed from the analysis six congruent items in which the cloze probability of the target word differed by more than .2 between the negated and the non-negated version of the item. These items violated the assumption that the cloze probability of the noun before the adjective is seen is (almost) the same in both levels of the informativity manipulation.
} 
cloze test. A further 49 stories were used in the incongruent condition. In these stories, the target noun was selected by the experimenter such that it was implausible, often anomalous, and its grammatical gender was not carried by any of the nouns proposed in the cloze test. Across two levels of informativity, the congruent nouns had a mean cloze probability of .2 (SD .21) and a constraint strength of .47 (SD .21). All incongruent nouns had a cloze probability of 0 and a mean constraint strength of .59 (SD .26; see Figure 3 for distributions).

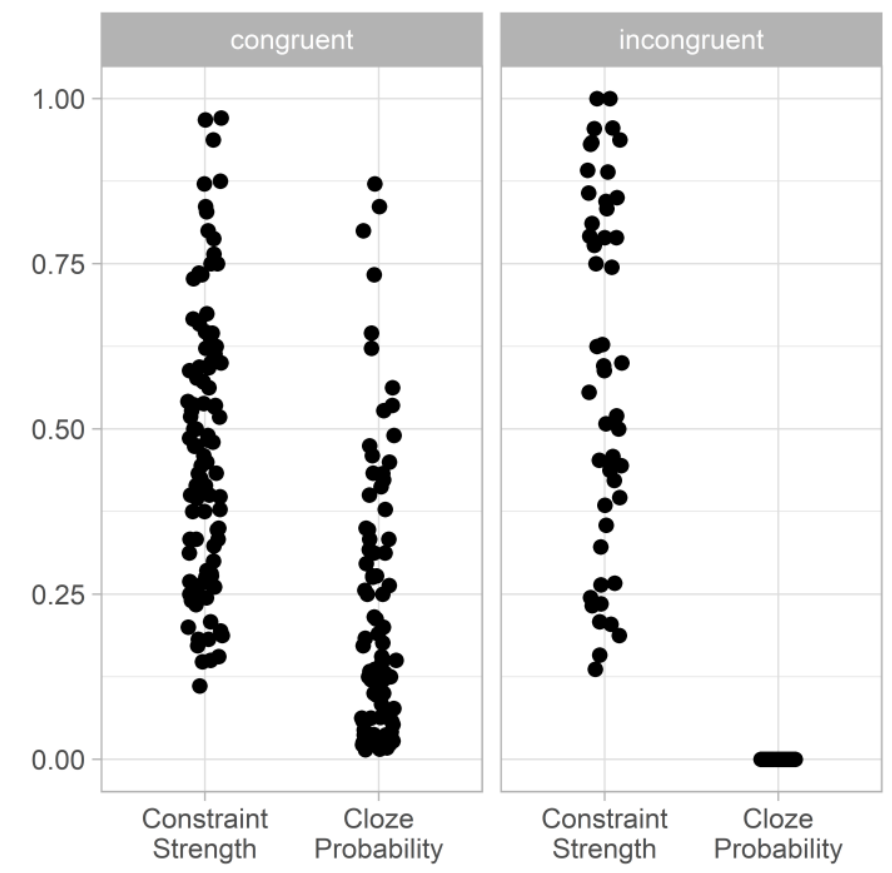

Figure 3. Constraint strength and cloze probability values for nouns in the congruent and incongruent items. These values are derived directly from cloze tests and are not updated by the adjectival suffix.

In all 146 items, the target noun was preceded by an adjective selected by the experimenter such that it could plausibly describe all the cloze test completions proposed for a given item. Next, two variants of each item were generated: more and less informative. Informativity was manipulated by either alternating the number feature of the direct object (singular vs. plural) or alternating the grammatical case of the direct object (genitive vs. accusative, attained via negating or not negating the verb). The choice of the specific manipulation used in each item was determined in the item selection procedure outlined above. In sum, the case was manipulated in 40 congruent and 25 incongruent items, whereas the number feature was manipulated in 57 congruent and 24 incongruent items. When the number feature was manipulated, care was taken to ensure that all cloze completions could plausibly occur in both the singular and plural. When the grammatical case was manipulated by negating the preceding verb, it was done to ensure that the direct objects that were predictable in the nonnegated version were also valid in the negated version (e.g. ... and he delivered ..., was changed to ... but surprisingly, he did not deliver ...). 
As indicated in the Introduction, for the statistical analyses we converted to surprisal the probability of the adjectival suffix and the updated probability of the noun. For each item, we also computed noun entropy before and after the adjective. Figure 4a shows how, for each item, the surprisal of the adjectival suffix and the updated surprisal of the noun changed as a result of the informativity manipulation in congruent items. Figure $4 \mathrm{~b}$ shows the relationship between adjectival suffix surprisal and noun entropy after the adjective.
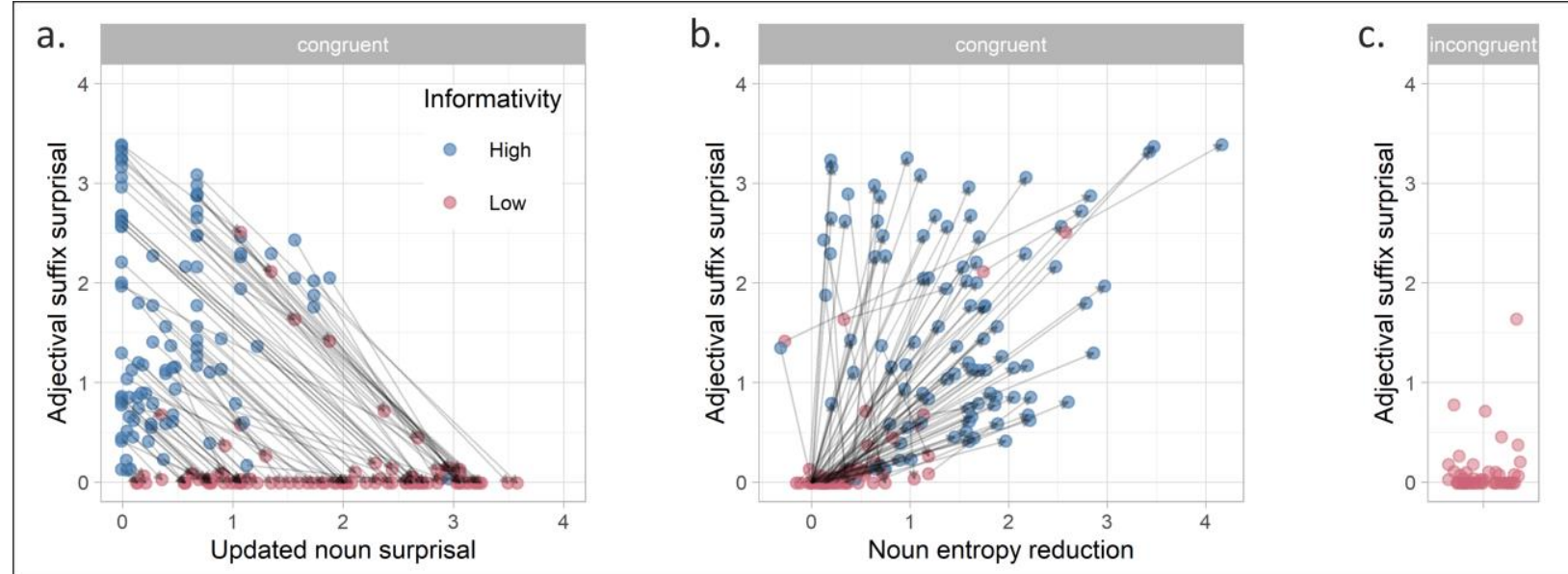

Figure 4. Panel A. Congruent items: the relationship between adjectival suffix surprisal and updated noun surprisal. Panel B. Congruent items: the relationship between adjectival suffix surprisal and noun entropy reduction. Panel C. Incongruent items: The distribution of adjectival suffix surprisal in the less informative condition. No more data for incongruent items is shown because of indefinite values for suffix surprisal (more informative condition) and for entropy reduction (both less and more informative condition).

Two lists of items were created, each of which contained all items: half in the more informative condition and half in the less informative condition. The more and the less informative conditions were counterbalanced across the two lists and across participants. In addition to the experimental items, we used 6 filler items that had the same structure as the experimental items; the critical nouns were congruent with the story and served as buffers following breaks in the experiment.

\section{Procedure}

Participants were seated in a dimly-lit, sound-attenuated room. Stimuli were presented on a 24" TFT screen in white letters on a dark grey background. Participants were seated approximately $90 \mathrm{~cm}$ from the screen. Each item was presented in two parts. The first part was presented all at once and consisted of all the story's sentences except the final one. This part remained on the screen until the participant pressed the spacebar key, indicating that s/he had finished reading the sentences. After the spacebar press, the screen went blank for $1300 \mathrm{~ms}$. After the blank period, a fixation marker ("+++") was presented in the center of the screen for 
$500 \mathrm{~ms}$, followed by a $500 \mathrm{~ms}$ blank, and then the word-by-word presentation of the final sentence. Each word was displayed for $300 \mathrm{~ms}$, centered in the middle of the screen, followed by a $200 \mathrm{~ms}$ blank interval. $1500 \mathrm{~ms}$ after the offset of the last word of the final sentence, presentation of the lead-in sentences of the next trial started. During the presentation of the storyfinal sentence, some content words were merged with adjacent function words. This was meant to facilitate reading by avoiding presentation of very short function words alone. Prepositions were displayed together with the word following them; similarly, reflexive verbs were displayed together with the reflexive pronoun ("się"). The critical words (the adjective and the noun of the direct object) were always displayed without any accompanying words. The number of display frames between the onset of the story final sentence and the target noun varied across items (mean 5.3, range 1-11, SD 2.0), so the position of the target words within the final sentence was not predictable.

Before the main part of the experiment, 4 training items were presented. Their structure was identical to the experimental items and they contained the congruent direct objects in the story-final sentence. After the training, participants were asked to blink their eyes for a few seconds and to shift their gaze between the crosses presented in the corners of the screen for a few seconds. This short procedure was meant to provide eye-movement EEG data that was subsequently used to facilitate establishment of eye-movement-related ICA components (see below). After each block of 50 items, participants were offered a break. Across participants, each of the list of experimental items was presented in four pseudo-randomized orders, with the only restriction that each break was followed by two filler items. The complete experiment (including electrode placement and removal and an off-line recall test) lasted approximately 80 minutes.

Participants were instructed to read all items carefully, to remain still, and to avoid eye movements and blinks in the period between the presentation of the fixation mark and the end of the story-final sentence. They were also told that after the experiment they would have to perform a sentence recall test. The test was introduced to ensure that the participants maintained their attention while reading the stories. The recall test consisted of 30 story-final sentences taken from the experimental items (20 congruent). All words were intact in the test sentences, but the direct object noun was blanked. Participants were asked to fill in the word that occurred in the blank position.

\section{EEG Recording}

EEG was recorded from 32 scalp sites by means of $\mathrm{AgAgCl}$ active electrodes (BioSemi Active-Two system) mounted in an elastic electrode cap (Electrocap International) at the standard 10-20 system locations. Recordings were referenced to the $\mathrm{C} 1$ electrode and rereferenced off-line to the mean of the left and right mastoids. The vertical EOG was monitored with two electrodes placed below and above a participant's right eye, and horizontal EOG was recorded from electrodes at the outer canthi of the eyes. The recordings were digitized online at $256 \mathrm{~Hz}$. Before segmentation, EEG was filtered off-line with a high-pass filter $(0.2 \mathrm{~Hz})$ to exclude slow signal drifts. 
Epochs from the continuous EEG in the interval between -200 and 800 ms with respect to the onset of the adjective and the noun were averaged and analyzed. Both adjective and noun used the baseline from the $200 \mathrm{~ms}$ preceding the adjective. Systematic artifacts resulting from eye movements, blinks and motor artifacts were filtered out using a procedure based on ICA (Delorme, Sejnowski, \& Makeig, 2007; Jung et al., 2000) run on 1-Hz-filtered data. Segments on electrodes that produced artifacts (skin potentials, poor electrode contact, etc.) were rejected using a logistic regression-based algorithm trained on manually marked artifacts. On average, $8.8 \%$ of trials were rejected, equally distributed across the experimental conditions. When an artifact occurred on one target word only, the segment on that electrode was nevertheless rejected on both the adjective and the noun.

Due to input lag resulting from using a thin-film transistor (TFT) display, stimuli were displayed $9 \mathrm{~ms}$ after the EEG trigger was sent and reached full brightness after 14ms (SD 0.4 $\mathrm{ms}$ ). To compensate for this lag, all EEG triggers were shifted 3 sampling points forward (11.7 $\mathrm{ms})$.

\section{Statistical analyses}

Two word positions were analyzed separately for congruent and incongruent items: the prenominal adjective and the target noun. At the adjectives in incongruent items, based on the prediction effect for similar incongruent items used in a previous study (Szewczyk \& Schriefers, 2013), we expected that the manipulation of informativity would lead to the N400 effect occurring on the parieto-occipital electrodes (PO3, PO4, O1, Oz, and O2) in the 375-575ms time window ${ }^{7}$. By extension, we speculated that a similar effect would occur for adjectives in congruent items. To anticipate, the effect at adjectives in the congruent condition resulted in a qualitatively different ERP effect than in incongruent items (i.e. the two effects occurred in two different time windows and partially non-overlapping electrodes); this forced us to analyze the effects in congruent and incongruent items separately. To statistically describe this novel effect that occurs in congruent items, we ran a non-parametric cluster-based permutation test (using $\mathrm{R}$ functions modelled on the algorithm described by Maris \& Oostenveld, 2007). The test confirmed a positivity at the adjective and identified a broadly distributed cluster (involving electrodes C3, C4, CP1, CP2, CP5, CP6, Cz, F3, F7, FC1, FC2, FC5, FC6, P3, P7, P8, Pz, T7, $\mathrm{T} 8$ ) in the $175-275 \mathrm{~ms}$ time window ( $<.05$, controlled for family-wise error rate). To conduct analyses parallel to those for incongruent adjectives and for nouns, we used averaged amplitudes from the selected cluster. However, note that this step of analysis is exploratory: cluster-based permutation tests can confirm the presence of a significant effect while controlling for familywise error rate, but they cannot unambiguously determine its time-course and scalp distribution (Maris \& Oostenveld, 2007).

\footnotetext{
${ }^{7}$ This time window and electrodes were established by running a series of LME models on the data from the Szewczyk and Schriefers (2013) study, binned into $25 \mathrm{~ms}$ intervals for each electrode and bin separately. We tested the effect of congruity (fixed effect and random slopes), looking for clusters of electrodes in which the effect of congruity remained significant for the longest time window (uncorrected for multiple comparisons).
} 
For the nouns, we were interested in modulations of the standard N400 component. Based on prior literature and effects obtained on similar items in our lab, we measured the N400 in the 300-450ms time window at the centro-parietal electrodes (CP1, CP2, P3, Pz, P4), both for congruent and incongruent items.

All results were analyzed at trial level using linear mixed effects models with participants and items as random variables using the lme4 package for $\mathrm{R}$ (Bates et al., 2015). As the dependent variable we used the mean amplitude in the selected electrodes and time windows. All continuous predictors were centered, while all categorical predictors were deviation coded. The actual fixed effects used in each model will be introduced before the respective analyses. We always used the maximal random effects structure with correlations between random effects removed. In all analyses we looked into the effect of trial number (and its interactions with other variables) to explore the possibility that prediction-related effects were not present at the onset of the study and only emerged as the experiment unfolded. In all analyses, we treated as significant effects with |t-value| greater than 2.

We conducted two types of analyses: with a factorial manipulation of Informativity and with continuous indices of Informativity.

Factorial manipulation of adjectival informativity. In this analysis, we fitted a statistical model with the same structure to the adjective and to the noun, for both congruent and incongruent items. The model consisted of fixed effects: Informativity (more and less informative variant of the item), Trial Number, and the interaction of Informativity and Trial Number.

Continuous indices of adjectival informativity. The aim of this set of analyses was to substitute the binary factor Informativity with more fine-grained factors that might better predict the engagement of prediction updating mechanisms: Suffix surprisal and Entropy reduction (noun entropy before the adjective minus noun entropy after the adjective; in this way, a greater value indicates a larger reduction). In addition, in the analyses on congruent nouns the fixed effects included the Noun Surprisal factor (derived from cloze probabilities computed before the adjective). All fixed effects were also tested in an interaction with Trial Number. This analysis will be conducted only on congruent items because Suffix surprisal and Entropy reduction is indefinite for more informative adjectives in incongruent items.

\section{Sentence recall test}

\section{Results}

On average, participants correctly recalled 54\% of target words (SD 11\%, range 27$77 \%)$. One participant with a score lower than $30 \%$ (27\%) was removed from the analysis. 


\section{Adjectives in congruent items}

Factorial manipulation of adjectival informativity. Figure 5 (panel 1A) shows ERPs time-locked to congruent adjectives in the less and more informative condition (see also Figure S1 in the Supplementary Materials, which shows ERPs to adjectives in the congruent and incongruent items together), while Figure 5 (panel 1B) shows the scalp distribution of t-values for the comparison between more and less informative conditions in the time window corresponding to the cluster identified by the permutation tests (175-275ms). The map reveals that adjectives with more informative suffixes led to more positive ERPs, compared to adjectives with less informative suffixes. The effect was strongest on centro-parietal electrodes. Despite the fact that visually the predictions of the LME model may suggest that the effect of Informativity is absent at the beginning of the experiment (see Figure 5, panel 1C), the interaction of Trial Number and Informativity was not significant (see Table 2).

Continuous indices of adjectival informativity. As can be seen in Table 3 , the main effect of Suffix surprisal was statistically significant. Overall, adjectives whose suffixes are surprising led to more positive ERPs within the cluster than adjectives with less surprising suffixes. There were no interactions with Trial number, indicating no support for the notion that the magnitude of the Suffix surprisal effect changed during the experiment.

Table 2

Estimates of fixed and random effects in the LME models using factorial manipulation of adjectival informativity in Experiment 1.

\begin{tabular}{|c|c|c|c|c|c|c|c|c|c|c|}
\hline \multirow[b]{2}{*}{ Effect } & \multicolumn{5}{|c|}{ Adjectives } & \multicolumn{5}{|c|}{ Nouns } \\
\hline & Estimate & SE & $\mathrm{t}$ & $\begin{array}{l}\text { by- } \\
\text { Item } \\
\text { SD }\end{array}$ & $\begin{array}{c}\text { by- } \\
\text { Subject } \\
\text { SD }\end{array}$ & Estimate & SE & $\mathrm{t}$ & $\begin{array}{l}\text { by- } \\
\text { Item } \\
\text { SD }\end{array}$ & $\begin{array}{c}\text { by- } \\
\text { Subject } \\
\text { SD }\end{array}$ \\
\hline \multicolumn{11}{|c|}{ Congruent items } \\
\hline Intercept & 1.72 & 0.28 & 6.16 & 1.36 & 1.28 & -0.39 & 0.25 & -1.57 & 1.84 & 0.27 \\
\hline TrialN & -0.03 & 0.04 & -0.9 & & 0.11 & 0.04 & 0.04 & 1.03 & & \\
\hline Informativity & 1.12 & 0.25 & 4.4 & 0.67 & & 1.22 & 0.4 & 3.06 & 1.69 & 0.96 \\
\hline TrialN:Informativity & 0.08 & 0.06 & 1.32 & & & 0.07 & 0.08 & 0.91 & & \\
\hline \multicolumn{11}{|c|}{ Incongruent items } \\
\hline Intercept & -2.19 & 0.31 & -7.06 & 0.96 & 1.26 & -4.44 & 0.39 & -11.49 & 0.98 & 1.68 \\
\hline TrialN & 0.04 & 0.05 & 0.91 & 0.03 & 0.12 & 0.02 & 0.07 & 0.24 & 0.22 & 0.16 \\
\hline Informativity & -1.08 & 0.49 & -2.19 & & 1.95 & 1.53 & 0.46 & 3.3 & & 0.33 \\
\hline TrialN:Informativity & -0.08 & 0.1 & -0.82 & & 0.34 & 0.04 & 0.13 & 0.33 & 0.32 & 0.32 \\
\hline
\end{tabular}

Note. Congruent items: 3,293 data-points; 97 items; 37 participants. Incongruent items: 1,658 data-points; 49 items; 37 participants. 


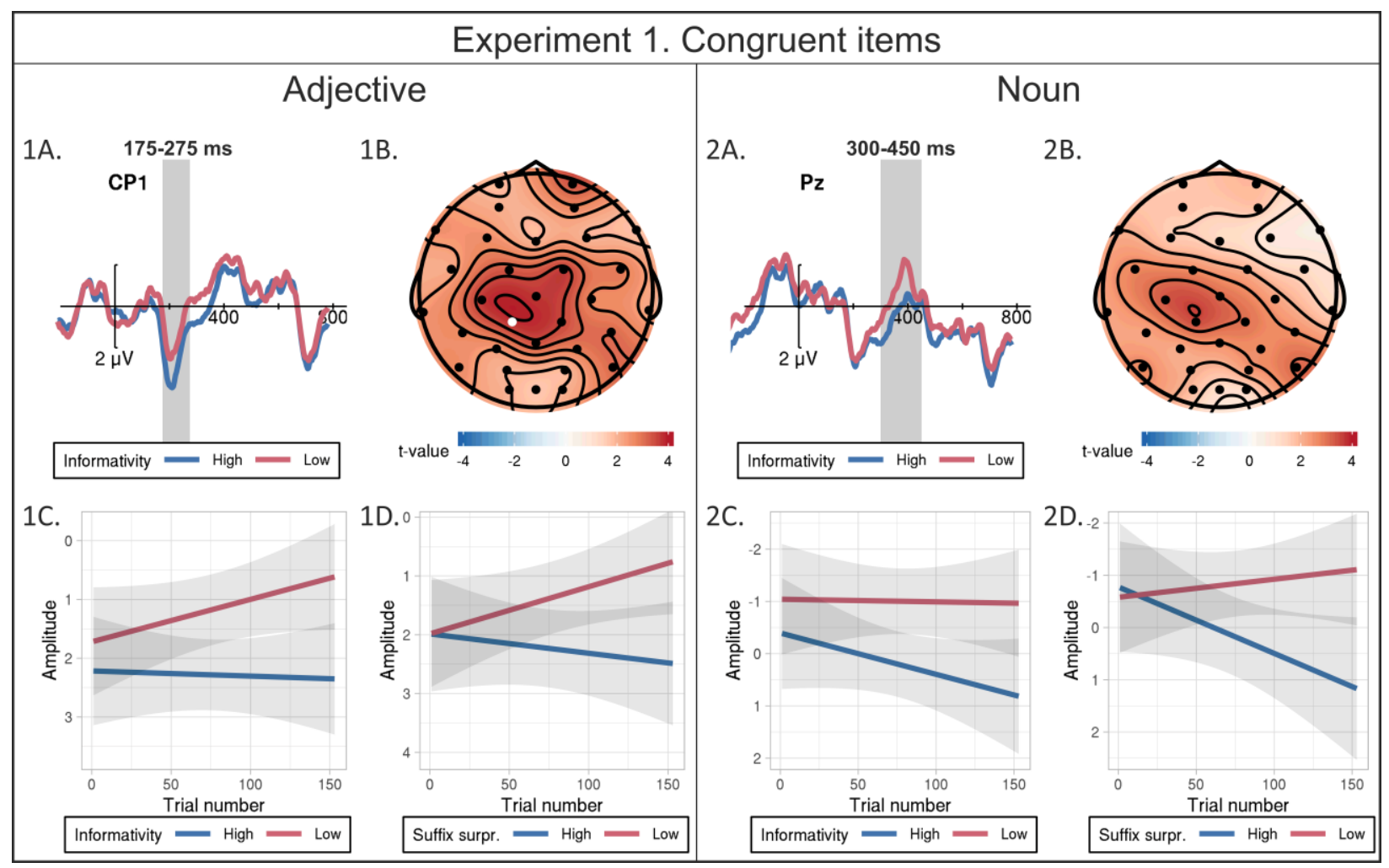

Figure 5. Panel 1A and 2A: Data-based ERPs time-locked to more and less informative adjectives (1A) or nouns (2A) in congruent items of Experiment 1. The shaded area delineates the time window of the analysis. Negative is up. Panel 1B and 2B: scalp distribution and t-values at each channel for the Informativity effect. Panels 1C and 2C: model-based estimates for high and low levels of Informativity as a function of Trial number. Panels 1D and 2D: model-based estimates for Suffix surprisal (median split) as a function of Trial number. Ribbons represent 95\% confidence bands. Panels 1 (A, B, C, D) show data for the adjective; panels 2 (A, B, C, D) show data for the noun.

\section{Congruent nouns}

Factorial manipulation of adjectival informativity. The analysis of the N400 component on congruent nouns revealed that nouns following more informative adjectives led to a reduction of the $\mathrm{N} 400$ component, relative to the same nouns following less informative adjectives (see Table 2 and Figure 5, panel 2A). The effect of Informativity-driven N400 reduction seems to be present across all trials.

Continuous indices of adjectival informativity. The analysis using continuous indices of adjectival informativity revealed significant effects of Noun surprisal and Suffix surprisal. Because Noun surprisal is based on pre-adjectival cloze values, while Suffix surprisal is assumed to index the amount of updating that the noun receives, these two combined effects reflect the updated surprisal of the noun. Neither effect was modified by Trial number (see Table 3), 
although descriptively the effect of Suffix surprisal appeared to be absent at the onset of the experiment (see Figure 5, panel 2D).

\section{Adjectives in incongruent items}

Factorial manipulation of adjectival informativity. For incongruent adjectives, i.e. adjectives which in the informative variant imply that no predictable nouns will occur, more informative adjectives led to a negativity. Both visually and based on statistics the effect was present across all trials (see Table 2 and Figure 6, panel 1C). For this analysis, the time window and electrodes were selected a priori based on the effect found at adjectives in the study by Szewczyk and Schriefers (2013). To test whether in the present study the effect had a similar time-course and scalp distribution, we ran the same exploratory procedure as described in Footnote 7. It revealed a cluster in the 400-525 time window on parietal electrodes (CP5, P3, P7, PO3) which was similar but not identical to the cluster found in our preceding study (Szewczyk $\&$ Schriefers, 2013; the effect was more anterior and left lateralized here).

Table 3

Estimates of fixed and random effects in the LME models using continuous indices of adjectival informativity in Experiment 1 (congruent items only).

\begin{tabular}{|c|c|c|c|c|c|c|c|c|c|c|}
\hline \multirow[b]{2}{*}{ Effect } & \multicolumn{5}{|c|}{ Adjectives } & \multicolumn{5}{|c|}{ Nouns } \\
\hline & Estimate & SE & $\mathrm{t}$ & $\begin{array}{l}\text { by- } \\
\text { Item } \\
\text { SD }\end{array}$ & $\begin{array}{c}\text { by- } \\
\text { Subject } \\
\text { SD }\end{array}$ & Estimate & SE & $\mathrm{t}$ & $\begin{array}{c}\text { by- } \\
\text { Item } \\
\text { SD }\end{array}$ & $\begin{array}{c}\text { by- } \\
\text { Subject } \\
\text { SD }\end{array}$ \\
\hline & \multicolumn{10}{|c|}{ Congruent items } \\
\hline Intercept & 1.74 & 0.28 & 6.23 & 1.35 & 1.28 & -0.39 & 0.24 & -1.62 & 1.69 & 0.25 \\
\hline TrialN & -0.03 & 0.04 & -0.87 & & 0.11 & 0.04 & 0.04 & 0.87 & & \\
\hline Suffix Surprisal & 0.52 & 0.2 & 2.63 & 0.32 & 0.51 & 0.63 & 0.31 & 2.05 & 0.85 & 0.51 \\
\hline Entropy Reduction & 0.15 & 0.23 & 0.64 & 0.36 & 0.53 & 0.05 & 0.31 & 0.15 & 0.01 & \\
\hline TrialN:Suffix Surprisal & 0.07 & 0.04 & 1.8 & - & & 0.1 & 0.06 & 1.62 & - & \\
\hline TrialN:Entropy Reduction & -0.06 & 0.04 & -1.33 & - & & -0.03 & 0.06 & -0.53 & - & \\
\hline Noun Surprisal & - & - & - & - & - & -0.83 & 0.24 & -3.39 & - & 0.28 \\
\hline TrialN:Noun Surprisal & - & - & - & - & - & -0.06 & 0.05 & -1.16 & - & 0.13 \\
\hline
\end{tabular}




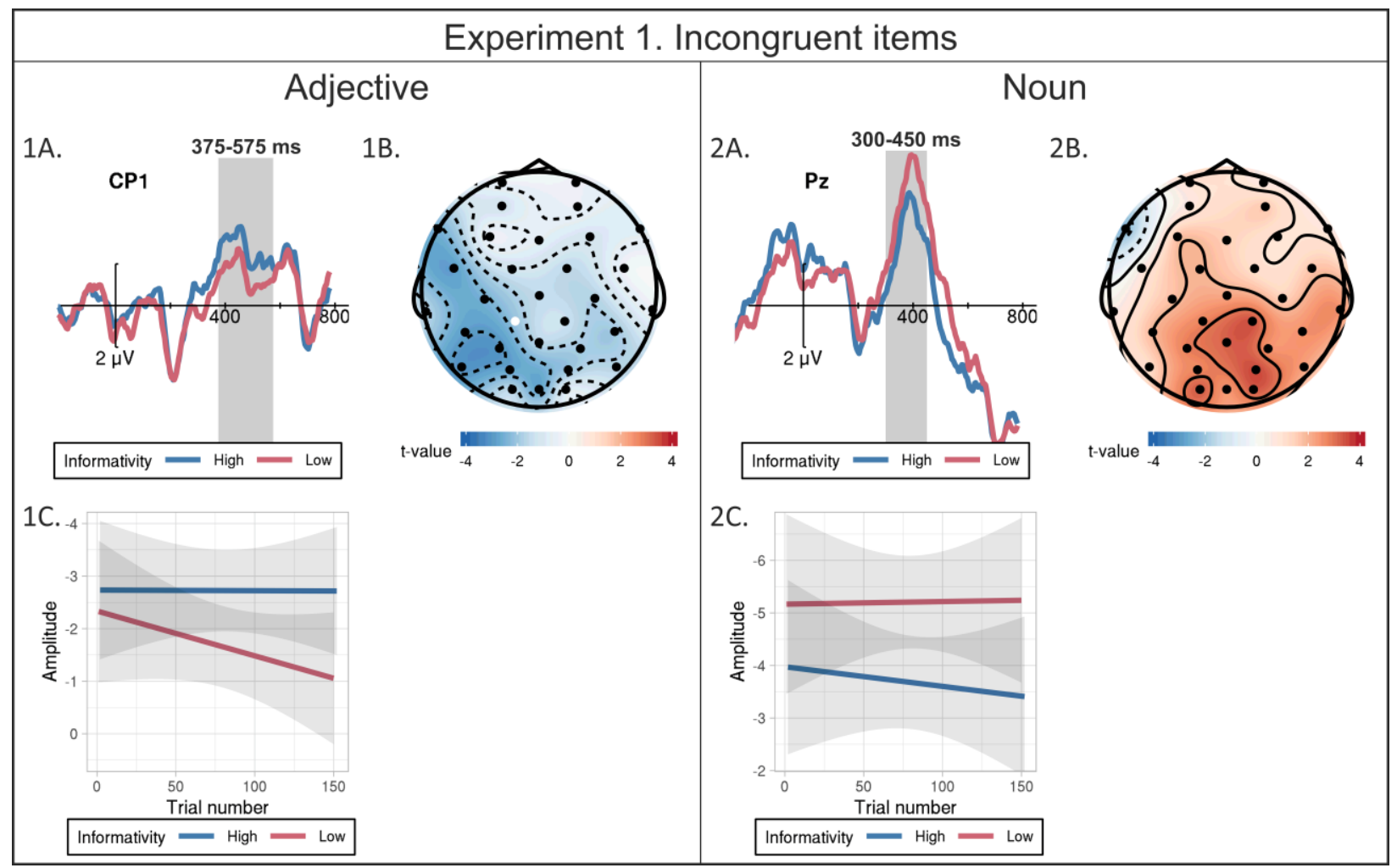

Figure 6. Panel 1A and 2A: Data-based ERPs time-locked to more and less informative adjectives (1A) or nouns (2A) in incongruent items of Experiment 1. The shaded area delineates the time window of the analysis. Negative is up. Panel 1B and 2B: scalp distribution and t-values at each channel for the Informativity effect. Panel 1C and 2C: model-based estimates for high and low levels of Informativity as a function of Trial number. Ribbons represent $95 \%$ confidence bands. Panels 1 (A, B, C) show data for the adjective; panels 2 (A, B, C) show data for the noun.

\section{Incongruent nouns}

Factorial manipulation of adjectival informativity. All incongruent nouns elicited a sizable negativity in the N400 time window (compare panels 2A of Figures 5 and 6); however, nouns following more informative adjectives elicited a smaller (less negative) N400 component relative to nouns following less informative adjectives. Both visually and based on statistics, the amplitude of the effect did not depend on Trial Number and appeared to be of constant magnitude across the entire experiment (see Table 2 and Figure 6, panel 2C).

\section{Post-hoc analyses}

In this study, adjectival informativity was manipulated in two ways: we changed the number feature of the direct object (single vs. plural) or we negated the main verb in the storyfinal sentence, which affected the grammatical case in which the direct object had to occur (positive verb: accusative case; negated verb: genitive case). The manipulation of the number feature does not require any change in the context, and thus may be considered a cleaner version of the informativity manipulation as compared to the grammatical case manipulation (which 
involves negating the main verb). To make sure that the effect of Informativity holds irrespective of the way in which Informativity was manipulated, we introduced a factorial manipulation of informativity with the inclusion of a deviation-coded predictor Manipulation Type (2 levels: number feature, grammatical case) and the interaction of Informativity and Manipulation Type. Statistical adjustment for the type of manipulation was meant to ensure that any significant effect of Informativity also holds at each level of Manipulation Type. The inclusion of the Manipulation Type factor did not improve the fit of any model. The details of the model comparison procedure and its results are reported in the Supplementary Materials (Table S1).

\section{Discussion}

In the current experiment we tested if ERP effects occurring on a gender-marked adjective that precedes a noun lead to updating predictions about the noun. If they do, we expected to find a reduced N400 to the noun when it is preceded by a more informative adjective (an adjective whose suffix more unambiguously indicates the gender of the noun) compared to when the noun is preceded by a less informative adjective. We expected to find this effect in congruent items but not necessarily in incongruent nouns. Nevertheless, we found support for the updating of predictions in both types of items: the N400 to nouns following more informative adjectives was reduced regardless of whether the noun was congruent or not.

We also hypothesized that the updating occurs at the adjective and it is reflected by an N400 effect whose amplitude is proportional to the amount of updating. The results only partially confirmed the hypothesis. We obtained an N400 effect for more vs. less informative adjectives but only in incongruent items. However, at adjectives in congruent items we found a novel and surprising effect: a positivity for more vs. less informative adjectives. Because of the novelty of the effect, we attempted to replicate Experiment 1.

Experiment 2 included also an exploratory analysis of the role of verbal fluency as an individual difference factor mediating the degree to which participants engage in generating predictions. Previous research showed that in older adults the ability to engage in making predictions may depend on verbal fluency (DeLong et al., 2012; Federmeier et al., 2010; Wlotko, Federmeier, \& Kutas, 2012). While in the current study we tested young adults, it is possible that prediction updating triggered by morphosyntactic information depends on individual differences in verbal fluency also in younger adults, a possibility that has never been tested before. 


\section{Experiment 2 \\ Methods}

\section{Participants}

The number of participants for Experiment 2 was established using a stopping rule. We collected data until the precision of the measurement of the effect of Informativity at adjectives in congruent items had improved by more than $25 \%$ (precision defined as $1 / \mathrm{SE}$ ). We ended up recruiting 48 participants. Participants were recruited using the same methods and from the same sources as in Experiment 1. Data from two participants were discarded (one due to a technical failure during the experiment, one due to an extremely low score in the recall test), leaving 46 participants for the analysis (mean age 22.2, SD 3.6, range 18.9-41.4). No participants reported having experienced a neurologic trauma or had any neurologic impairment. Thirty-seven participants were right-handed, and among them twelve reported left-handed relatives in their immediate family.

\section{Materials}

We used the same materials as in Experiment 1.

\section{Procedure}

We followed the same procedure as in Experiment 1 . The only difference was that we also conducted semantic category and letter fluency tasks before and after the main task. For the letter fluency tasks we used the letters M and B; for the category fluency we used "body parts" and "animal names" categories, with the order of the tasks counterbalanced across the participants. For the analyses, fluencies before and after the task were averaged. A small fraction $(3.7 \%)$ of fluencies were lost due to a technical error.

\section{EEG Recording}

The EEG was recorded in the same way as in Experiment 1. The EEG preprocessing was also the same, but this time we used a weaker high-pass filter $(0.1 \mathrm{~Hz})$. Moreover, instead of subtracting the baseline, we used it as a predictor in the regression analyses, a procedure that greatly improves the precision of estimates of ERP effects (Alday, 2019) ${ }^{8}$. To this aim, the regression models contain a new fixed effect Baseline. Artifacts were removed using the same procedure and algorithm as in Experiment 1. On average, $10.4 \%$ of trials were rejected, equally distributed across the experimental conditions.

\section{Statistical analyses}

We used the same analysis strategy as in Experiment 1. For the confirmatory analysis on adjectives in congruent items, we used the cluster identified via exploratory analysis in Experiment 1.

${ }^{8}$ The overall pattern of results in Experiment 1 and 2 did not depend on these differences in high-pass filters or on the way baseline was taken into account. 


\section{Sentence recall test}

\section{Results}

On average, participants correctly recalled $56 \%$ of target words (SD 13\%, range 7-77\%). One participant with a score lower than $30 \%$ (7\%) was removed from the analysis.

\section{Adjectives in congruent items}

Factorial and continuous manipulation of adjectival informativity. The analysis did not replicate the positivity reported in Experiment 1 for the comparison between the more and less informative conditions (see Table 4). As can be seen in Figure 7 (panel 1A), the ERPs for the two conditions are on top of each other. Moreover, cluster-based permutation tests did not reveal any other variable that would affect the ERPs to the adjectives (Suffix surprisal, Entropy reduction). In summary, no variable that we controlled for reliably predicted EEG modulations at adjectives at any scalp location or time window.

Table 4

Estimates of fixed and random effects in the LME models using factorial manipulation of adjectival informativity in Experiment 2.

\begin{tabular}{|c|c|c|c|c|c|c|c|c|c|c|}
\hline \multirow[b]{2}{*}{ Effect } & \multicolumn{5}{|c|}{ Adjectives } & \multicolumn{5}{|c|}{ Nouns } \\
\hline & Estimate & SE & $\mathrm{t}$ & $\begin{array}{l}\text { by- } \\
\text { Item } \\
\text { SD }\end{array}$ & $\begin{array}{c}\text { by- } \\
\text { Subject } \\
\text { SD }\end{array}$ & Estimate & SE & $\mathrm{t}$ & $\begin{array}{c}\text { by- } \\
\text { Item } \\
\text { SD }\end{array}$ & $\begin{array}{c}\text { by- } \\
\text { Subject } \\
\text { SD }\end{array}$ \\
\hline \multicolumn{11}{|c|}{ Congruent items } \\
\hline Intercept & 0.71 & 0.22 & 3.2 & 0.76 & 1.25 & -2.53 & 0.32 & -7.93 & 1.57 & 1.65 \\
\hline Baseline & 6.89 & 0.17 & 40.87 & & 0.89 & 5.77 & 0.29 & 19.74 & 0.88 & 1.59 \\
\hline TrialN & 0 & 0.03 & 0 & 0.04 & 0.09 & 0.09 & 0.04 & 2.55 & 0.11 & 0.07 \\
\hline Informativity & 0 & 0.19 & 0.01 & & & 1.15 & 0.29 & 3.95 & 1.24 & 0.38 \\
\hline TrialN:Informativity & -0.05 & 0.05 & -0.98 & & 0.21 & -0.08 & 0.07 & -1.16 & 0 & 0.09 \\
\hline \multicolumn{11}{|c|}{ Incongruent items } \\
\hline Intercept & -3.52 & 0.24 & -14.75 & 0.84 & 1.03 & -5.82 & 0.45 & -12.85 & 1.5 & 2.39 \\
\hline Baseline & 7.32 & 0.24 & 30.25 & 0.66 & 1.07 & 5.64 & 0.33 & 17.14 & 1.1 & 1.37 \\
\hline TrialN & 0 & 0.03 & -0.15 & 0.03 & & 0.05 & 0.05 & 1.01 & 0.11 & 0.16 \\
\hline Informativity & -1 & 0.28 & -3.6 & & & 1.29 & 0.47 & 2.73 & & 1.96 \\
\hline TrialN:Informativity & 0.02 & 0.09 & 0.21 & 0.27 & 0.31 & -0.01 & 0.09 & -0.1 & & 0.17 \\
\hline
\end{tabular}

Note: Congruent items: 4082 data-points; 97 items; 46 participants. Incongruent items: 2026 data-points; 49 items; 46 participants. 


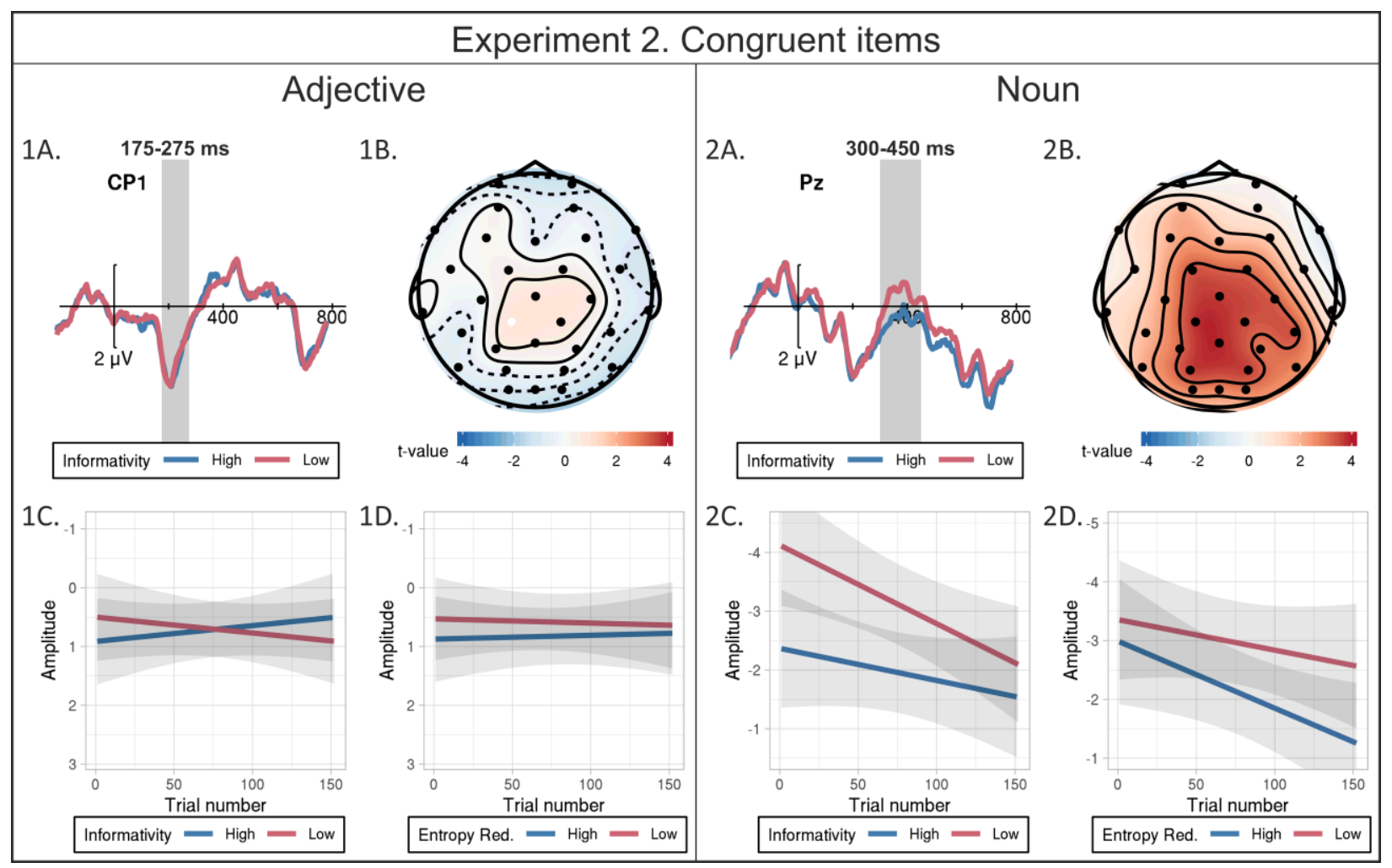

Figure 7. Panel 1A and 2A: Data-based ERPs time-locked to more and less informative adjectives (1A) or nouns (2A) in congruent items of Experiment 2. The shaded area delineates the time window of the analysis. Negative is up. Panel 1B and 2B: scalp distribution and t-values at each channel for the Informativity effect. Panels 1C and 2C: model-based estimates for high and low levels of Informativity as a function of Trial number. Panels 1D and 2D: model-based estimates for Entropy Reduction (median split) as a function of Trial number. Ribbons represent 95\% confidence bands. Panels 1 (A, B, C, D) show data for the adjective; panels 2 (A, B, C, D) show data for the noun.

\section{Congruent nouns}

Factorial manipulation of adjectival informativity. The analysis replicated the reduction of the N400 component on congruent nouns following more informative adjectives compared to less informative adjectives (see Table 4 and Figure 7, panels 2A and 2C). As in Experiment 1, the effect of Informativity-driven N400 reduction seemed to be present across all trials.

Continuous indices of adjectival informativity. The results of Experiment 1 were partially replicated. As in the previous experiment, Noun surprisal (based on cloze values measured before the adjective) co-determined the amplitude of the N400 to the noun. Unlike in Experiment 1, the adjectival update was mediated by Entropy reduction rather than Suffix surprisal (see Table 5 and Figure 7, panel 2D). Neither effect was modulated by a significant interaction with Trial. 


\section{Adjectives in incongruent items}

Factorial manipulation of adjectival informativity. As can be seen in Figure 8 (panel 1A) and Table 4, more informative adjectives led to a centro-parietal negativity (a late N400 effect) compared to less informative adjectives, thus fully replicating Experiment 1 and the effect found by Szewczyk and Schriefers (2013). The data do not support the idea that the size of the effect changed as the experiment unfolded.

Table 5

Estimates of fixed and random effects in the LME models using continuous indices of adjectival informativity in Experiment 2 (congruent items only).

\begin{tabular}{|c|c|c|c|c|c|c|c|c|c|c|}
\hline \multirow[b]{2}{*}{ Effect } & \multicolumn{5}{|c|}{ Adjectives } & \multicolumn{5}{|c|}{ Nouns } \\
\hline & Estimate & SE & $\mathrm{t}$ & $\begin{array}{l}\text { by- } \\
\text { Item } \\
\text { SD }\end{array}$ & $\begin{array}{c}\text { by- } \\
\text { Subject } \\
\text { SD }\end{array}$ & Estimate & SE & $\mathrm{t}$ & $\begin{array}{l}\text { by- } \\
\text { Item } \\
\text { SD }\end{array}$ & $\begin{array}{c}\text { by- } \\
\text { Subject } \\
\text { SD }\end{array}$ \\
\hline & \multicolumn{10}{|c|}{ Congruent items } \\
\hline Intercept & 0.7 & 0.22 & 3.17 & 0.78 & 1.26 & -2.54 & 0.32 & -8 & 1.5 & 1.65 \\
\hline Baseline & 6.9 & 0.17 & 40.84 & & 0.89 & 5.77 & 0.29 & 19.85 & 0.89 & 1.57 \\
\hline TrialN & 0 & 0.03 & 0.01 & 0.05 & 0.09 & 0.08 & 0.04 & 2.25 & 0.13 & 0.06 \\
\hline Suffix Surprisal & -0.06 & 0.13 & -0.51 & & & 0.03 & 0.21 & 0.14 & & \\
\hline Entropy Reduction & 0.17 & 0.15 & 1.16 & & & 0.61 & 0.25 & 2.42 & 0.56 & 0.36 \\
\hline TrialN:Suffix Surprisal & -0.02 & 0.03 & -0.66 & - & & -0.09 & 0.05 & -1.68 & - & 0.13 \\
\hline TrialN:Entropy Reduction & -0.01 & 0.03 & -0.29 & - & 0.07 & 0.04 & 0.05 & 0.87 & - & \\
\hline Noun Surprisal & - & - & - & - & - & -0.5 & 0.2 & -2.55 & - & \\
\hline TrialN:Noun Surprisal & - & - & - & - & - & 0.05 & 0.04 & 1.22 & - & \\
\hline
\end{tabular}




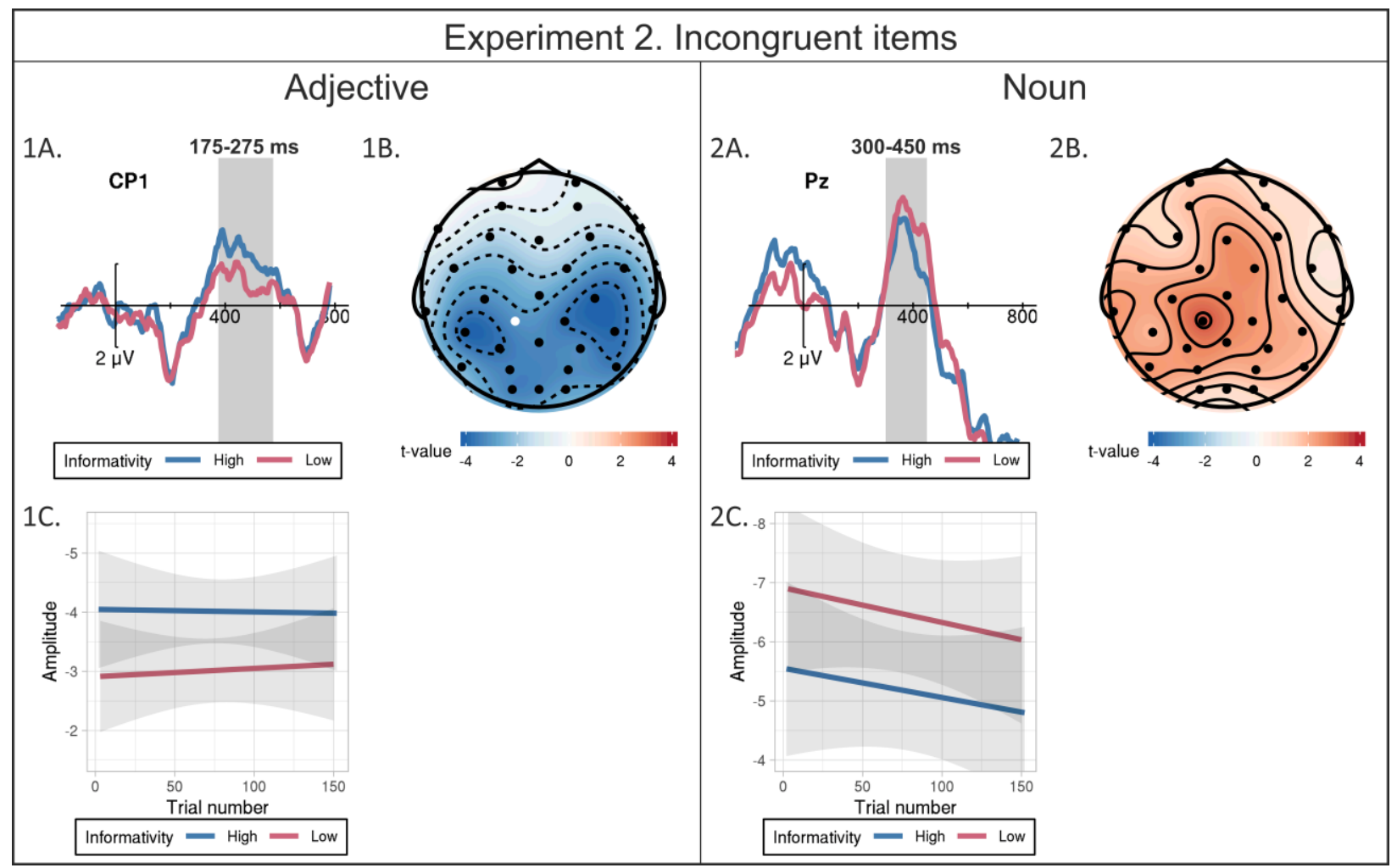

Figure 8. Panel 1A and 2A: Data-based ERPs time-locked to more and less informative adjectives (1A) or nouns (2A) in incongruent items of Experiment 2. The shaded area delineates the time window of the analysis. Negative is up. Panel 1B and 2B: scalp distribution and t-values at each channel for the Informativity effect. Panels 1C and 2C: model-based estimates for high and low levels of Informativity as a function of Trial number. Ribbons represent $95 \%$ confidence bands. Panels 1 (A, B, C) show data for the adjective; panels 2 (A, B, C) show data for the noun.

\section{Incongruent nouns}

Factorial manipulation of adjectival informativity. As expected, incongruent nouns in both conditions elicited a large N400 effect (compare panel 2A in Figures 7 and 8; see also Figure S5 in the Supplementary Materials), but the N400 for nouns following more informative adjectives was smaller (less negative) compared to nouns following less informative adjectives. As in the replicated experiment, the effect of informativity was constant across the entire experiment (see Table 4 and Figure 8, panel 2C).

\section{Post-hoc analyses}

Type of informativity manipulation. As in Experiment 1, we checked if the inclusion of the interaction between Manipulation Type and Informativity improved the fit of any of the above models. We found that it did not, confirming the robustness of the Informativity effect to the method with which it was manipulated (see Table S1 in the Supplementary Materials). 


\section{Verbal fluency.}

Letter and semantic category fluency did not interact with Informativity in any of the models, indicating no support for the notion that young adults with better verbal fluency are more likely to update their predictions in a timely fashion (see Table S2 in the Supplementary Materials).

\section{Discussion}

The primary aim of Experiment 2 was to replicate Experiment 1 and in particular the unexpected effect of positivity at adjectives in congruent items. Although most of the effects found in Experiment 1 replicated, there were two notable differences between the outcomes of the two experiments. The first difference was that in Experiment 2 we did not replicate the Informativity effect at adjectives in congruent items: more and less informative adjectives led to almost exactly the same ERP responses. On the other hand, we still observed a reduction of the N400 effect at congruent nouns following more vs. less informative adjectives. The second difference concerned the nature of the N400 reduction at congruent nouns. In the analysis using continuous predictors of adjectival informativity, we found that the reduction of the N400 component was mediated by adjective-induced reduction of noun entropy rather than surprisal with the adjectival suffix. We provide a more in-depth discussion of the findings in the General Discussion. 


\section{General Discussion}

One of the strongest arguments for prediction in language comprehension comes from studies that manipulate whether a prenominal word (e.g. a determiner or an adjective) matches a highly predictable noun (DeLong et al., 2012, 2005; Foucart et al., 2014; Martin et al., 2013; Otten et al., 2007; Otten \& Van Berkum, 2008, 2009; Van Berkum et al., 2005; Wicha et al., 2004) or a set of nouns (Szewczyk \& Schriefers, 2013). These studies show that presentation of a prenominal word that mismatches the predictable word leads to a differential ERP reaction relative to when the prenominal word matches the predictable word. This ERP reaction indicates that the predictable noun must indeed have been predicted. However, the functional role of the mechanism that triggers the negativity at the prenominal word is unknown. One possibility is that this effect reflects the mismatch between the noun and the prenominal word (Dikker \& Pylkkänen, 2013). As an alternative explanation, we have proposed that such effects reflect the updating of predictions about the noun (Szewczyk, 2016; Szewczyk \& Schriefers, 2018). More specifically, we proposed that the updating is realized through changing the activation level of representations in LTM, which is manifested in an increased amplitude of the N400 component on the adjective.

To test the prediction updating hypothesis, native speakers of Polish read short stories that made several nouns likely at the position of a direct object in the story's final sentence. Our design employed two types of items: congruent and incongruent. In congruent items, one of the likely nouns was actually used in the story as the critical noun (mean cloze probability $=.2$; see Figure 3 for distribution). In incongruent items, the critical noun was not predictable and did not semantically fit the preceding context. In all items, the critical noun was preceded by an adjective which always agreed in gender with the following noun.

Critically, we manipulated informativity of the prenominal adjective about the upcoming noun. In congruent items, more informative adjectives indicated that some predictable nouns could not follow the adjective because they would not match the gender of the adjective, thereby increasing the likelihood that the critical noun which matched the gender of the adjective would follow. In incongruent items, more informative adjectives indicated that none of the predictable nouns could follow because none of them matched the adjective's gender. The adjectives also occurred in a less informative variant in which - thanks to the syncretisms of Polish adjectival inflection - the gender marking at the adjective was ambiguous and agreed with more than one grammatical gender. Thus, in the congruent items the less informative adjectives matched a larger set of predictable nouns, thus promoting the critical congruent noun to a lesser extent (compared to the more informative condition). In the incongruent items, adjectives in the less informative variant concealed the fact that an incongruent noun would arrive because the gender marking at the adjective also matched some predictable nouns. Importantly, the same adjective and noun were used in the more and in the less informative variant of each item and they differed only in their grammatical form (grammatical case or number feature). In Polish, the gender of 
adjectives is reflected by their suffix, so the information necessary for prediction updating was encoded in the adjectival suffix.

We hypothesized that if the ERP effect on the prenominal adjectives reflects prediction updating, the effect's size in microvolts should be related to the informativity of the gender suffix at the adjective. Consequently, the N400 effect at the noun, which reflects the degree of preactivation of the noun, should be reduced proportionally to the informativity of the preceding adjective. Most of the results confirmed these predictions. Just as expected, when the noun was preceded by a more informative adjective, relative to when it was preceded by a less informative adjective, the N400 to the noun was reduced both for congruent and incongruent items. Also, in line with our predictions, ERPs to adjectives in incongruent items were modulated by the informativity of the adjective, thus replicating our previous findings (Szewczyk \& Schriefers, 2013). Our predictions were not confirmed with regards to the adjectives in congruent items, and the two experiments provided different results for these items. In Experiment 1, more informative adjectives in congruent items led to a positivity compared to less informative adjectives. However, in Experiment 2, we failed to replicate this effect, finding no differences in the ERPs between more and less informative adjectives for congruent items. Later in the General Discussion, we will return to the possible sources of this divergence between the experiments.

\section{Did processing the adjectives lead to updating predictions about the upcoming nouns?}

The prediction updating hypothesis started from the question of whether ERP effects found in studies using the "prenominal words" paradigm (see Introduction) indeed reflect prediction updating about an upcoming noun. The only way to address such a question is to test whether predictions about the upcoming noun indeed get updated as a result of processing the prenominal word. In the current study, the prenominal words were adjectives. To see whether the processing of adjectives really results in prediction updating, we looked at N400s at subsequent nouns. We reasoned that if a noun becomes predicted to a higher degree, then, by definition, its activation should increase. In consequence, when the noun is displayed, it should elicit an N400 with reduced amplitude. To check whether this is the case, we compared N400s to nouns following more informative adjectives (which afforded more prediction updating) against the same nouns following less informative adjectives (which afforded less prediction updating). We found that in the case of both congruent and incongruent items the N400 for nouns following more informative adjectives was reduced compared to nouns following less informative adjectives. This effect was robust in both the experiments we conducted. This strongly suggests that the information introduced at the adjective led to prediction updating.

We also showed that the effect arises independently of how informativity was manipulated (grammatical case vs. number feature). Because the same noun (and adjective) was used in the more and less informative variant of each item, the reduction of the $\mathrm{N} 400$ at the noun must be a consequence of apprehending the information provided by the suffix of the preceding adjective, thus confirming the prediction updating hypothesis. We will now focus on the question of what process(es) led to prediction updating at adjectives. 


\section{Mechanisms of prediction updating}

To understand the specific mechanisms used to update predictions, we will focus on the ERP effects elicited by the adjectives. Because the pattern of ERP effects at adjectives differed between congruent and incongruent items, we will discuss them separately.

\section{Prediction updating in incongruent items}

The results for adjectives in incongruent items followed our predictions and replicated the N400 reported in our previous study (Szewczyk \& Schriefers, 2013). The N400 for incongruent items in Experiment 1 and 2, as well as the N400 in our previous study overlapped to a large extent spatially and temporally. In the current study, the effect was elicited by more informative adjectives whose form unambiguously indicated that none of the already predicted words could be the upcoming head noun ${ }^{9}$.

Within the prediction updating hypothesis (see Introduction), we proposed that at the moment when the adjective is encountered in a constraining context, a set of direct object nouns has already been preactivated in long-term memory. In the course of processing the adjective, the information about the adjective's gender is accessed and gets automatically matched with the gender of all the preactivated nouns. We proposed that when none of the nouns matches the gender of the adjective, a new set of nouns gets preactivated, leading to the N400 at the adjective. In other words, the information encoded in the gender marking of the adjective is causally responsible for facilitating access to the noun.

Strong support for this claim is provided by the finding that nouns that follow more informative adjectives elicited N400 with reduced amplitude. At the same time, this finding rules out an alternative explanation of the effects at prenominal adjectives in incongruent items, namely that the ERP effect on the adjective simply reflects noticing the gender mismatch with the predicted nouns. The current results show that the effect at adjectives goes beyond merely noticing the mismatch and reflects preactivation of possible upcoming nouns using the new information that is provided by the adjectival suffix.

More broadly, this finding is in line with previous studies showing that when a word $\mathrm{X}$ preactivates a following word Y (e.g. a verb preactivates its possible arguments), the processing of the word $\mathrm{Y}$ is facilitated whereas the processing of the word $\mathrm{X}$ is associated with a greater cost. The processing cost is reflected either by increased N400 amplitude (Freuenberger \& Roehm, 2017; Maess et al., 2016; Ness \& Meltzer-Asscher, 2018) or by increased activity in the middle temporal gyrus (Fruchter et al., 2015; see also Chow \& Che, 2020 for evidence from eyetracking). The present study extends those findings by demonstrating a causal connection

\footnotetext{
${ }^{9}$ In our previous studies (Szewczyk \& Schriefers, 2013; 2018) we referred to the N400 as 'delayed' because its latency seemed to be shifted by about $50 \mathrm{~ms}$. When we explicitly compared the latency of the $\mathrm{N} 400$ at adjectives and nouns in this study, we found reliable delays of mean peak latency of $24 \mathrm{~ms}$ and $13 \mathrm{~ms}$ (for Experiment 1 and 2, respectively). This delay may occur simply because N400s on adjectives occur later than on nouns (this might be responsible for the 20-30 ms delay of the N400 in the antonym condition in Kutas \& Iragui, 1998). It may also be caused by the possibility that the first-pass sweep through LTM at the adjectives needs more time to reach possible nouns' representations because it is mediated by accessing the gender of the adjective, which is necessary to resolve the agreement between the adjective and potential nouns.
} 
between the increased N400 amplitude at the word X and the reduced N400 at the word Y. Moreover, in the previous studies it was the semantics of the word $\mathrm{X}$ that was associated with the facilitated processing at the word $\mathrm{Y}$, whereas in the current study, the informative cue was encoded in a morphosyntactic feature of the word X.

It is noteworthy that in our study participants had no way of predicting that the actually presented incongruent target noun would follow the adjective. More informative adjectives indicated only the gender of the noun, while the semantic context provided no clue about the meaning of the noun because the noun was incongruent. The only way to explain why access to the incongruent noun was facilitated anyway is to assume that the suffix of more informative adjectives (which unambiguously indicated the gender of the noun phrase) led to the preactivation of all nouns carrying that gender, irrespective of their semantic fit to the context. This is an unexpected result because the prediction updating hypothesis assumes that the adjective can trigger preactivation of words that match the gender of the adjective and fit the context semantically. One reason for such a result might be that in this study the grammatical gender of the adjective was a more reliable cue about the upcoming noun than the preceding semantic context. This is because the gender agreement between the noun and the adjective was never violated, whereas semantic congruity between the noun and the preceding context was not maintained in $31 \%$ of items. It is possible that participants adapted to this pattern and when the two types of cues conflicted, they favored the morphosyntactic cue.

It is also possible that the prediction of the whole gender class of nouns was mediated via animacy. Most of the incongruent target nouns (88\%) carried the masculine-animate or masculine-inanimate gender, so it could be possible that the gender suffix of informative adjectives weakly preactivated animate (for masculine-animate suffixes) or inanimate (for masculine-inanimate suffixes) nouns. To test this possibility, further research will have to include a higher proportion of incongruent items using nouns with the feminine or neuter genders, namely gender classes that are not associated with semantic animacy or inanimacy.

\section{Prediction updating in congruent items}

Based on the prediction updating hypothesis, we hypothesized that the informativity manipulation in congruent items would show the same type of effect as in incongruent items. More specifically, we expected that the manipulation of adjectival informativity would result in a modulation of the N400 to the adjective and in a modulation of the N400 on the subsequent noun. The results only partly confirmed our hypothesis. While nouns following more informative adjectives indeed elicited a reduced N400 component compared to nouns following less informative adjectives, the ERPs to the adjectives did not align with our hypothesis: they either showed an early positivity for more informative items (Experiment 1), or no ERP modulation at all (Experiment 2). Thus, on the one hand we confirmed that in congruent items adjectives do induce prediction updating about upcoming nouns (as evidenced by the reduction of the N400 at the noun), but on the other hand, we did not establish a clear ERP pattern associated with prediction updating at the adjective. Clearly, whatever the mechanism of prediction updating at 
the adjective in congruent items was, it was different from the mechanism employed in incongruent items ${ }^{10}$.

Retrospectively, the existence of two mechanisms of prediction updating may be unsurprising. In incongruent items, the adjectival suffix did not match any of the predictable nouns, so here prediction updating could consist in activating completely new nouns (as explained above and as proposed by the prediction updating theory). In contrast, in the case of congruent items the adjectival suffixes always matched in gender some of the predictable nouns, therefore there was no need to preactivate new nouns. Instead, the information introduced by the adjectival suffix could be used to reconfigure the activation strength of the already preactivated nouns, namely to increase the activation of the nouns matching in gender the suffix of the adjective and perhaps also to deactivate nouns that were not compatible. At the computational level, such a mechanism could be described as Bayesian updating (Kuperberg \& Jaeger, 2016).

The existence of two different mechanisms of prediction updating would explain why the ERP indices of prediction updating at adjectives for congruent and incongruent items were different in Experiment 1, but this does not explain why there was no reflection of prediction updating in congruent items in Experiment 2 or why the Informativity manipulation led to a positivity vs. no effect in Experiment 1 and 2, respectively.

Whatever the reason for the difference between Experiments 1 and 2, the absence of any ERP effect at adjectives in congruent items in Experiment 2 does not necessarily mean that no computations involved in prediction updating occurred there. As already noted, some computations had to occur at the adjective because the amplitude of the N400 to the noun was reduced following more informative adjectives. One possibility is that the absence of any ERP effect was an artifact which simply resulted from sampling error. Another possibility is that the intensity of computations involved in prediction updating in congruent items was the same, regardless of the amount of prediction reconfiguration triggered by the adjective. Yet another possibility is that prediction updating was not strictly time-locked to the onset of the adjective and instead resulted in non-phase-locked modulations of power in some frequencies. Finally, the computations could occur in a region of the cortex that is only weakly reflected at the scalp (Koessler et al., 2009).

\section{Factors underlying informativity}

In addition to the tests based on the categorical Informativity factor, for congruent items we also conducted analyses using more fine-grained and continuous indices of information content drawn from Information Theory. We contrasted two ways in which prediction updating

\footnotetext{
${ }^{10}$ It could be argued that because different stories were used in the congruent and incongruent items, the lexical properties of the adjectives could lead to diverging effects at the adjectives. However, we think this is rather unlikely for the following reasons: 1) there is no obvious mechanism in which lexical properties of adjectives would interact with the informativity manipulation; 2) over half the adjectives used in the incongruent items were also used in the congruent items; 3) ERPs to adjectives in the less informative condition were identical for congruent and incongruent items (see Figures S1 and S3 in the Supplementary Materials). If there were relevant differences in the lexical properties of the adjectives, they should also surface in the less informative conditions.
} 
could be measured in congruent items: adjectival suffix surprisal (which is equal to the change in noun surprisal thanks to learning about the noun's gender from the adjectival suffix) and the reduction in noun entropy. In congruent items, the relevance of the two indices of information content was different across the experiments. In Experiment 1, both the positivity at the adjective and the reduction of $\mathrm{N} 400$ at the noun were best accounted for by suffix surprisal. In Experiment 2, however, neither manipulation accounted for variance in the ERPs to the adjective, whereas the reduction of the $\mathrm{N} 400$ at the noun was best predicted by entropy reduction. Speculatively, this difference between the best predictor of the N400 amplitude at congruent nouns in Experiment 1 and 2 could indicate that different strategies or processes underlie prediction updating in the two experiments. In consequence, these different strategies/processes could be linked to the presence of the effect at the preceding adjectives. However, at this point it is impossible to determine whether the difference in results between the experiments for congruent items was due to chance or some differences in processing strategy.

\section{Properties of predictions and the prediction updating}

The data obtained in the two experiments permit formulation of some more general conclusions about the mechanisms of prediction in sentence comprehension and prediction updating.

\section{Predictions are parallel}

We were the first to experimentally demonstrate that when reading sentences comprehenders preactivate multiple words in parallel (Szewczyk \& Schriefers, 2013). More recently, this idea has gained some traction as other researchers have provided convergent evidence (Morgan et al., 2018). Here we provide two novel arguments in favor of this claim. The first one is based on the fact that the N400 amplitude for incongruent nouns was reduced following more informative adjectives. This indicates that thousands of nouns matching the gender of the informative adjective must have been preactivated as a result of processing the adjectival suffix. This is a very strong version of parallel predictions.

The second argument is based on the comparison between congruent and incongruent items. More informative adjectives in the incongruent items are the only condition in which we unambiguously know that whatever predictions a participant had the adjectival suffix disconfirmed them. Such adjectives elicited an N400 effect. Conversely, in the congruent items the suffix always matched the gender of some of the predictable nouns. Because the majority of the congruent items made several nouns predictable, if participants predicted only one noun at a time, their predictions should have been inaccurate most of the time. The probability of encountering an adjectival suffix that violates prediction should increase when the suffix is improbable. In these situations - as we know from incongruent items - the adjectives should lead to a delayed N400. However, in congruent items we saw a different pattern: the less likely adjectival suffixes led to either a positivity (Experiment 1) or no effect (Experiment 2). This pattern is incompatible with the idea that readers predict only one word at a time, while it is fully 
compatible with the idea of updating parallel predictions - as already envisaged in the Introduction.

\section{Prediction updating is unlikely to be an adaptation to experimental items}

In ERP studies on sentence comprehension, participants encounter texts that are very different from natural language; this might alter the strategy with which they process the sentences. In some experiments, reading short stories that change topic from item to item may discourage them from formulating context-based predictions. On the other hand, when critical words occur in the same repetitive context (e.g. always in a direct object of the story-final sentence) and they occasionally contain semantic violations, after some time participants may realize that the critical words are "special" and start to sense when the critical words are likely to be encountered. Such awareness may help participants decide when engaging in or updating predictions might be worth the effort (see Brothers, Swaab, \& Traxler, 2017; Lau, Holcomb, \& Kuperberg, 2013; Wlotko \& Federmeier, 2015 for demonstrations of how context can affect predictive processing). Such altered strategies may limit the extent to which we can generalize results of experiments to everyday language processing because they only demonstrate adaptation to "prediction-encouraging experimental set-ups" (Huettig \& Mani, 2016). We tried to determine whether this was the case by looking at how the effects that reflect prediction updating evolved in time across each experiment. If the prediction updating effects reflect such adaptations and result from the repetitive structure of the items, then we would expect that prediction-updating effects should be absent at the beginning of the study and appear only later as participants familiarize themselves with the items.

The results do not support the idea that prediction updating is an adaptation to specific experimental items. None of the analyses in Experiment 1 or 2 showed any significant interaction between trial number and the indices of prediction updating. While this does not rule out that prediction updating may be modulated by recent experiences of the comprehender, to some extent it is unlikely that a special strategy or task set is a prerequisite for prediction updating to occur. As such, prediction updating seems to be a ubiquitous mechanism that supports language comprehension each time useful morphosyntactic cues are encountered.

\section{Conclusions}

Previous work has shown that readers predict words that are highly likely in a given context. We extend this work by showing that these existing predictions get updated when readers encounter an informative morphosyntactic cue. This causes facilitated processing of the cued noun, reflected by a reduced N400 component at the noun.

Prediction updating can be implemented using different mechanisms which are recruited depending on the compatibility of the cue with the existing predictions. We established that when the cue mismatches all predicted words, new nouns that match the cue get activated via a mechanism that is reflected by an N400. When the cue matches at least some predicted words, another mechanism is recruited to shift activation to nouns congruent with the cue. However, the 
second mechanism is not very well understood and more research is needed to elucidate its exact properties.

Overall, this study shows that sentence processing is a highly incremental process in which every informative piece of data is immediately used to support comprehension. This flexibility is possible thanks to the employment of different activation mechanisms that are recruited depending on a given cue. It is also possible due to cooperation between the different levels of representation (here, between conceptual and morphosyntactic representations). The actual (pre)activations are a result of overlapping constraints occurring at multiple levels (c.f. MacDonald, Pearlmutter, \& Seidenberg, 1994; Tanenhaus \& Trueswell, 1995). 
The mechanisms of prediction updating

\section{Acknowledgements}

This project was partially supported by a SONATA BIS grant awarded by the National Science Center Poland to Z.W. (Sonata BIS grant 015/18/E/HS6/00428). We would like to thank all members of the LangUsta Lab for helpful discussion at an early stage of this project, Marta Marecka, Weronika Browarczyk, and Mateusz Wojnarowicz for their help with data collection, and to Kara Federmeier for providing very helpful comments on an earlier version of this manuscript. 


\section{References}

Alday, P. M. (2019). How much baseline correction do we need in ERP research? Extended GLM model can replace baseline correction while lifting its limits. Psychophysiology. https://doi.org/10.1111/psyp.13451

Altmann, G. T. M., \& Kamide, Y. (1999). Incremental interpretation at verbs: Restricting the domain of subsequent reference. Cognition, 73(3), 247-264.

Bates, D., Mächler, M., Bolker, B., \& Walker, S. (2015). Fitting Linear Mixed-Effects Models using lme4. Journal of Statistical Software, 67(1), 1-48. https://doi.org/10.18637/jss.v067.i01

Brothers, T., Swaab, T. Y., \& Traxler, M. J. (2017). Goals and strategies influence lexical prediction during sentence comprehension. Journal of Memory and Language, 93, 203216. https://doi.org/10.1016/j.jml.2016.10.002

Brown, C., \& Hagoort, P. (1993). The processing nature of the N400: Evidence from masked priming. Journal of Cognitive Neuroscience, 5(1), 34-44.

Carter, B. T., Foster, B., Muncy, N. M., \& Luke, S. G. (2019). Linguistic networks associated with lexical, semantic and syntactic predictability in reading: A fixation-related fMRI study. NeuroImage, 189, 224-240. https://doi.org/10.1016/j.neuroimage.2019.01.018

Chow, W.-Y., \& Chen, D. (2020). Predicting (in)correctly: Listeners rapidly use unexpected information to revise their predictions. Language, Cognition and Neuroscience. Advance online publication. https://doi.org/10.1080/23273798.2020.1733627

Delaney-Busch, N., Morgan, E., Lau, E. F., \& Kuperberg, G. R. (2017). Comprehenders Rationally Adapt Semantic Predictions to the Statistics of the Local Environment: a Bayesian Model of Trial-by-Trial N400 Amplitudes. In G. Gunzelmann, A. Howes, T. Tenbrink, \& E. J. Davelaar (Eds.), Proceedings of the 39th Annual Conference of the Cognitive Science Society (pp. 283-288).

DeLong, K. A., Groppe, D. M., Urbach, T. P., \& Kutas, M. (2012). Thinking ahead or not? Natural aging and anticipation during reading. Brain and Language, 121(3), 226-239. https://doi.org/10.1016/j.bandl.2012.02.006

DeLong, K. A., Urbach, T. P., \& Kutas, M. (2005). Probabilistic word pre-activation during language comprehension inferred from electrical brain activity. Nature Neuroscience, 8(8), 1117-1121. https://doi.org/10.1038/nn1504

Delorme, A., Sejnowski, T., \& Makeig, S. (2007). Enhanced detection of artifacts in EEG data using higher-order statistics and independent component analysis. NeuroImage, 34(4), 1443-1449. https://doi.org/10.1016/j.neuroimage.2006.11.004

Dikker, S., \& Pylkkänen, L. (2013). Predicting language: MEG evidence for lexical preactivation. Brain and Language, 127(1), 55-64. https://doi.org/10.1016/j.bandl.2012.08.004

Duffy, S. A., Henderson, J. M., \& Morris, R. K. (1989). Semantic facilitation of lexical access during sentence processing. Journal of Experimental Psychology: Learning, Memory, and Cognition, 15(5), 791-801. https://doi.org/10.1037/0278-7393.15.5.791 
Ehrlich, S. F., \& Rayner, K. (1981). Contextual effects on word perception and eye movements during reading. Journal of Verbal Learning and Verbal Behavior, 20(6), 641-655. https://doi.org/10.1016/S0022-5371(81)90220-6

Federmeier, K. D., Kutas, M., \& Schul, R. (2010). Age-related and individual differences in the use of prediction during language comprehension. Brain and Language, 115(3), 149-161. https://doi.org/10.1016/j.bandl.2010.07.006

Foucart, A., Martin, C. D., Moreno, E. M., \& Costa, A. (2014). Can bilinguals see it coming? Word anticipation in L2 sentence reading. Journal of Experimental Psychology: Learning, Memory, and Cognition, 40(5), 1461-1469. https://doi.org/10.1037/a0036756

Frank, S. L., Otten, L. J., Galli, G., \& Vigliocco, G. (2013). Word surprisal predicts N400 amplitude during reading. Proceedings of the 51st Annual Meeting of the Association for Computational Linguistics, 878-883. Retrieved from http://anthology.aclweb.org/P/P13/P13-2.pdf\#page=926

Frank, S. L., \& Willems, R. M. (2017). Word predictability and semantic similarity show distinct patterns of brain activity during language comprehension. Language, Cognition and Neuroscience, 32(9), 1192-1203. https://doi.org/10.1080/23273798.2017.1323109

Freunberger, D., \& Roehm, D. (2017). The costs of being certain: Brain potential evidence for linguistic preactivation in sentence processing. Psychophysiology, 54(6), 824-832. https://doi.org/10.1111/psyp.12848

Fruchter, J., Linzen, T., Westerlund, M., \& Marantz, A. (2015). Lexical Preactivation in Basic Linguistic Phrases. Journal Of Cognitive Neuroscience, 27(10), 1912-1935. https://doi.org/10.1162/jocn_a_00822

Hale, J. (2006). Uncertainty About the Rest of the Sentence. Cognitive Science, 30(4), 643-672. https://doi.org/10.1207/s15516709cog0000_64

Henderson, J. M., Choi, W., Lowder, M. W., \& Ferreira, F. (2016). Language structure in the brain: A fixation-related fMRI study of syntactic surprisal in reading. NeuroImage, 132, 293-300. https://doi.org/10.1016/j.neuroimage.2016.02.050

Huettig, F., \& Mani, N. (2016). Is prediction necessary to understand language? Probably not. Language, Cognition and Neuroscience, 31(1), 19-31. https://doi.org/10.1080/23273798.2015.1072223

Jung, T.-P., Makeig, S., Humphries, C., Lee, T.-W., McKeown, M. J., Iragui, V., \& Sejnowski, T. J. (2000). Removing electroencephalographic artifacts by blind source separation. Psychophysiology, 37(2), 163-178. https://doi.org/10.1111/1469-8986.3720163

Kleiman, G. M. (1980). Sentence frame contexts and lexical decisions: Sentence-acceptability and word-relatedness effects. Memory \& Cognition, 8(4), 336-344. https://doi.org/10.3758/BF03198273

Kochari, A. R., \& Flecken, M. (2018). Lexical prediction in language comprehension: A replication study of grammatical gender effects in Dutch. Language, Cognition and Neuroscience, O(0), 1-15. https://doi.org/10.1080/23273798.2018.1524500 
Koessler, L., Maillard, L., Benhadid, A., Vignal, J. P., Felblinger, J., Vespignani, H., \& Braun, M. (2009). Automated cortical projection of EEG sensors: Anatomical correlation via the international 10-10 system. NeuroImage, 46(1), 64-72.

https://doi.org/10.1016/j.neuroimage.2009.02.006

Kuperberg, G. R., \& Jaeger, T. F. (2016). What do we mean by prediction in language comprehension? Language, Cognition and Neuroscience, 31(1), 32-59. https://doi.org/10.1080/23273798.2015.1102299

Kutas, M., \& Iragui, V. (1998). The N400 in a semantic categorization task across 6 decades. Electroencephalography and Clinical Neurophysiology/Evoked Potentials Section, 108(5), 456-471.

Kutas, M., DeLong, K. A., \& Smith, N. J. (2011). A look around at what lies ahead: Prediction and predictability in language processing. In M. Bar (Ed.), Predictions in the brain: Using our past to generate a future (pp. 190-207). Oxford University Press.

Kutas, M., \& Federmeier, K. D. (2011). Thirty Years and Counting: Finding Meaning in the N400 Component of the Event-Related Brain Potential (ERP). Annual Review of Psychology, 62(1), 621-647. https://doi.org/10.1146/annurev.psych.093008.131123

Lau, E. F., Holcomb, P. J., \& Kuperberg, G. R. (2013). Dissociating N400 effects of prediction from association in single word contexts. Journal of Cognitive Neuroscience, 25(3), 484502. https://doi.org/10.1162/jocn_a_00328

Lau, E. F., \& Namyst, A. (2019). FMRI evidence that left posterior temporal cortex contributes to N400 effects of predictability independent of congruity. Brain and Language, 199, 104697. https://doi.org/10.1016/j.bandl.2019.104697

Levy, R. (2008). Expectation-based syntactic comprehension. Cognition, 106(3), 1126-1177. https://doi.org/10.1016/j.cognition.2007.05.006

Linzen, T., \& Jaeger, T. F. (2015). Uncertainty and Expectation in Sentence Processing: Evidence From Subcategorization Distributions. Cognitive Science, 40(6), 1382-1411. https://doi.org/10.1111/cogs.12274

Lowder, M. W., Choi, W., Ferreira, F., \& Henderson, J. M. (2018). Lexical Predictability During Natural Reading: Effects of Surprisal and Entropy Reduction. Cognitive Science, 42(S4), 1166-1183. https://doi.org/10.1111/cogs.12597

MacDonald, M. C., Pearlmutter, N. J., \& Seidenberg, M. S. (1994). The lexical nature of syntactic ambiguity resolution. Psychological Review, 101(4), 676.

Maess, B., Mamashli, F., Obleser, J., Helle, L., \& Friederici, A. D. (2016). Prediction Signatures in the Brain: Semantic Pre-Activation during Language Comprehension. Frontiers in Human Neuroscience, 10. https://doi.org/10.3389/fnhum.2016.00591

Maris, E., \& Oostenveld, R. (2007). Nonparametric statistical testing of EEG- and MEG-data. Journal of Neuroscience Methods, 164(1), 177-190. https://doi.org/10.1016/j.jneumeth.2007.03.024

Martin, C. D., Thierry, G., Kuipers, J.-R., Boutonnet, B., Foucart, A., \& Costa, A. (2013). Bilinguals reading in their second language do not predict upcoming words as native 
readers do. Journal of Memory and Language, 69(4), 574-588.

https://doi.org/10.1016/j.jml.2013.08.001

McClelland, J. L., \& O’Regan, J. K. (1981). Expectations increase the benefit derived from parafoveal visual information in reading words aloud. Journal of Experimental Psychology: Human Perception and Performance, 7(3), 634. https://doi.org/10.1037/0096-1523.7.3.634

Morgan, E., Delaney-Busch, N., Kim, M., Warnke, L., Wlotko, E. W., \& Kuperberg, G. R. (2018). N400 evidence for parallel lexical predictions. Presented at the 31st Annual CUNY Sentence Processing Conference, Davis, CA.

Ness, T., \& Meltzer-Asscher, A. (2018). Predictive Preupdating and Working Memory Capacity: Evidence from Event-related Potentials. Journal of Cognitive Neuroscience. https://doi.org/10.1162/jocn_a_01322

Nieuwland, M. S., Politzer-Ahles, S., Heyselaar, E., Segaert, K., Darley, E., Kazanina, N., ... Huettig, F. (2018). Large-scale replication study reveals a limit on probabilistic prediction in language comprehension. ELife, 7, e33468. https://doi.org/10.7554/eLife.33468

Oldfield, R. C. (1971). The assessment and analysis of handedness: The Edinburgh inventory. Neuropsychologia, 9(1), 97-113. https://doi.org/10.1016/0028-3932(71)90067-4

Open Science Framework (2016). Szewczyk, J.: Two mechanisms of prediction updating [materials, data, scripts]. Retrieved from https://osf.io/c6hxt/. https://doi.org/10.17605/OSF.IO/C6HXT

Otten, M., Nieuwland, M. S., \& Van Berkum, J. J. A. (2007). Great expectations: Specific lexical anticipation influences the processing of spoken language. BMC Neuroscience, 8(1), 89. https://doi.org/10.1186/1471-2202-8-89

Otten, M., \& Van Berkum, J. J. A. (2008). Discourse-Based Word Anticipation During Language Processing: Prediction or Priming? Discourse Processes, 45(6), 464-496. https://doi.org/10.1080/01638530802356463

Otten, M., \& Van Berkum, J. J. A. (2009). Does working memory capacity affect the ability to predict upcoming words in discourse? Brain Research, 1291, 92-101. https://doi.org/10.1016/j.brainres.2009.07.042

Rayner, K., \& Well, A. D. (1996). Effects of contextual constraint on eye movements in reading: A further examination. Psychonomic Bulletin \& Review, 3(4), 504-509. https://doi.org/10.3758/BF03214555

Schuster, S., Hawelka, S., Hutzler, F., Kronbichler, M., \& Richlan, F. (2016). Words in Context: The Effects of Length, Frequency, and Predictability on Brain Responses During Natural Reading. Cerebral Cortex, 26(10), 3889-3904. https://doi.org/10.1093/cercor/bhw184

Schwanenflugel, P. J., \& LaCount, K. L. (1988). Semantic relatedness and the scope of facilitation for upcoming words in sentences. Journal of Experimental Psychology: Learning, Memory, and Cognition, 14(2), 344. https://doi.org/10.1037/02787393.14.2.344 
Schwanenflugel, P. J., \& Shoben, E. J. (1985). The influence of sentence constraint on the scope of facilitation for upcoming words. Journal of Memory and Language, 24(2), 232-252. https://doi.org/10.1016/0749-596X(85)90026-9

Schwanenflugel, P. J., \& White, C. R. (1991). The influence of paragraph information on the processing of upcoming words. Reading Research Quarterly, 26(2), 160-177. https://doi.org/10.2307/747980

Smith, N. J., \& Levy, R. (2013). The effect of word predictability on reading time is logarithmic. Cognition, 128(3), 302-319. https://doi.org/10.1016/j.cognition.2013.02.013

Szewczyk, J. M. (2016). The mechanisms of predictions in language comprehension Nijmegen: Radboud Unviersity.

Szewczyk, J. M., \& Schriefers, H. (2011). Is animacy special? ERP correlates of semantic violations and animacy violations in sentence processing. Brain Research, 1368, 208221. https://doi.org/10.1016/j.brainres.2010.10.070

Szewczyk, J. M., \& Schriefers, H. (2013). Prediction in language comprehension beyond specific words: An ERP study on sentence comprehension in Polish. Journal of Memory and Language, 68(4), 297-314. https://doi.org/10.1016/j.jml.2012.12.002

Szewczyk, J. M., \& Schriefers, H. (2018). The N400 as an index of lexical preactivation and its implications for prediction in language comprehension. Language, Cognition and Neuroscience, 33(6), 665-686. http://dx.doi.org/10.1080/23273798.2017.1401101

Tanenhaus, M. K., \& Trueswell, J. C. (1995). Sentence comprehension. In Handbook of Perception and Cognition: Vol. 11. Speech, language, and communication (2nd ed., pp. 217-262). https://doi.org/10.1016/B978-012497770-9.50009-1

Van Berkum, J. J. A., Brown, C. M., Zwitserlood, P., Kooijman, V., \& Hagoort, P. (2005). Anticipating Upcoming Words in Discourse: Evidence From ERPs and Reading Times. Journal of Experimental Psychology: Learning, Memory, and Cognition, 31(3), 443-467. https://doi.org/10.1037/0278-7393.31.3.443

Van Berkum, J. J. A., Hagoort, P., \& Brown, C. M. (1999). Semantic integration in sentences and discourse: Evidence from the N400. Journal of Cognitive Neuroscience, 11(6), 657671.

Wicha, N. Y. Y., Bates, E. A., Moreno, E. M., \& Kutas, M. (2003). Potato not Pope: Human brain potentials to gender expectation and agreement in Spanish spoken sentences. Neuroscience Letters, 346(3), 165-168. https://doi.org/10.1016/S0304-3940(03)00599-8

Wicha, N. Y. Y., Moreno, E. M., \& Kutas, M. (2003). Expecting gender: An event-related brain potential study on the role of grammatical gender in comprehending a line drawing within a written sentence in Spanish. Cortex, 39, 483-508.

Wicha, N. Y. Y., Moreno, E. M., \& Kutas, M. (2004). Anticipating words and their gender: An event-related brain potential study of semantic integration, gender expectancy, and gender agreement in Spanish sentence reading. Journal of Cognitive Neuroscience, 16(7), $1272-1288$. 
Willems, R. M., Frank, S. L., Nijhof, A. D., Hagoort, P., \& van den Bosch, A. (2016). Prediction During Natural Language Comprehension. Cerebral Cortex, 26(6), 2506-2516. https://doi.org/10.1093/cercor/bhv075

Wlotko, E. W., Federmeier, K. D., \& Kutas, M. (2012). To Predict or Not to Predict: AgeRelated Differences in the Use of Sentential Context. Psychology and Aging, 27(4), 975988. https://doi.org/10.1037/a0029206

Wlotko, E. W., \& Federmeier, K. D. (2015). Time for prediction? The effect of presentation rate on predictive sentence comprehension during word-by-word reading. Cortex, 68, 20-32. https://doi.org/10.1016/j.cortex.2015.03.014

Yan, S., Kuperberg, G. R., \& Jaeger, T. F. (2017). Prediction (Or Not) During Language Processing. A Commentary On Nieuwland et al. (2017) And Delong et al. (2005). BioRxiv, 143750. https://doi.org/10.1101/143750

Yun, J., Chen, Z., Hunter, T., Whitman, J., \& Hale, J. (2015). Uncertainty in processing relative clauses across East Asian languages. Journal of East Asian Linguistics, 24(2), 113-148. https://doi.org/10.1007/s10831-014-9126-6 


\section{Supplementary Materials}

\section{Suffix surprisal, preadjectival noun surprisal and updated noun surprisal}

In the main body, we claimed that when a congruent noun is preceded by an adjective with a matching gender suffix, surprisal of the noun is equal to the sum of surprisal of the noun without seeing the adjective and the surprisal of the adjective's gender.

We are interested in computing the probability of a noun conditioned by knowing its gender and the preceding semantic context: $p$ ( $N \mid$ gender, context).

From Bayes rules:

$$
\begin{array}{r}
p(N \mid \text { gender }, \text { context })=\frac{p(N \cap \text { gender } \cap \text { context })}{p(\text { gender } \cap \text { context })} \\
=\frac{p(\text { gender } \mid \text { noun }, \text { contex } t) * p(\text { noun } \cap \text { context })}{p(\text { gender } \cap \text { context })}
\end{array}
$$

We notice that $p$ (gender $\mid$ noun, context $)=1$ because if we know a noun, we also know the gender of the preceding adjective. This simplifies our formula to:

$$
\begin{aligned}
& p(N \mid \text { gender }, \text { context })=\frac{p(\text { noun } \cap \text { context })}{p(\text { gender } \cap \text { context })}=\frac{p(\text { noun } \mid \text { context }) * p(\text { contex })}{p(\text { gender } \mid \text { context }) * p(\text { context })} \\
& =\frac{p(\text { noun } \mid \text { context })}{p(\text { gender } \mid \text { context })}
\end{aligned}
$$

Next, we convert noun probability given gender and context into surprisal:

$$
\begin{array}{r}
-\log p(N \mid \text { gender }, \text { context })=-\log \left(\frac{p(\text { noun } \mid \text { context })}{p(\text { gender } \mid \text { context })}\right) \\
=-\log p(\text { noun } \mid \text { contex })+\log p(\text { gender } \mid \text { context })
\end{array}
$$

The first part of the resulting equation corresponds to surprisal of the noun before seeing the adjective. The second part of the equation corresponds to surprisal of the adjectival gender. In other words, knowing the gender of the noun reduces noun surprisal by the amount of surprisal induced by learning something about the gender of the noun.

The above formula holds as long as all nouns in a given item have a fully determined gender. This was the case in the majority of our items. A few exceptions were items containing nouns which currently undergo a linguistic change from one gender to another (most often from masculine-inanimate to masculine-animate). For those items, as updated surprisal of the noun we used values computed directly from the cloze tests, which reflected the current proportion of participants using a given noun in each of the genders. 
The mechanisms of prediction updating

\section{ERPs to adjectives in congruent and incongruent items in Experiment 1}

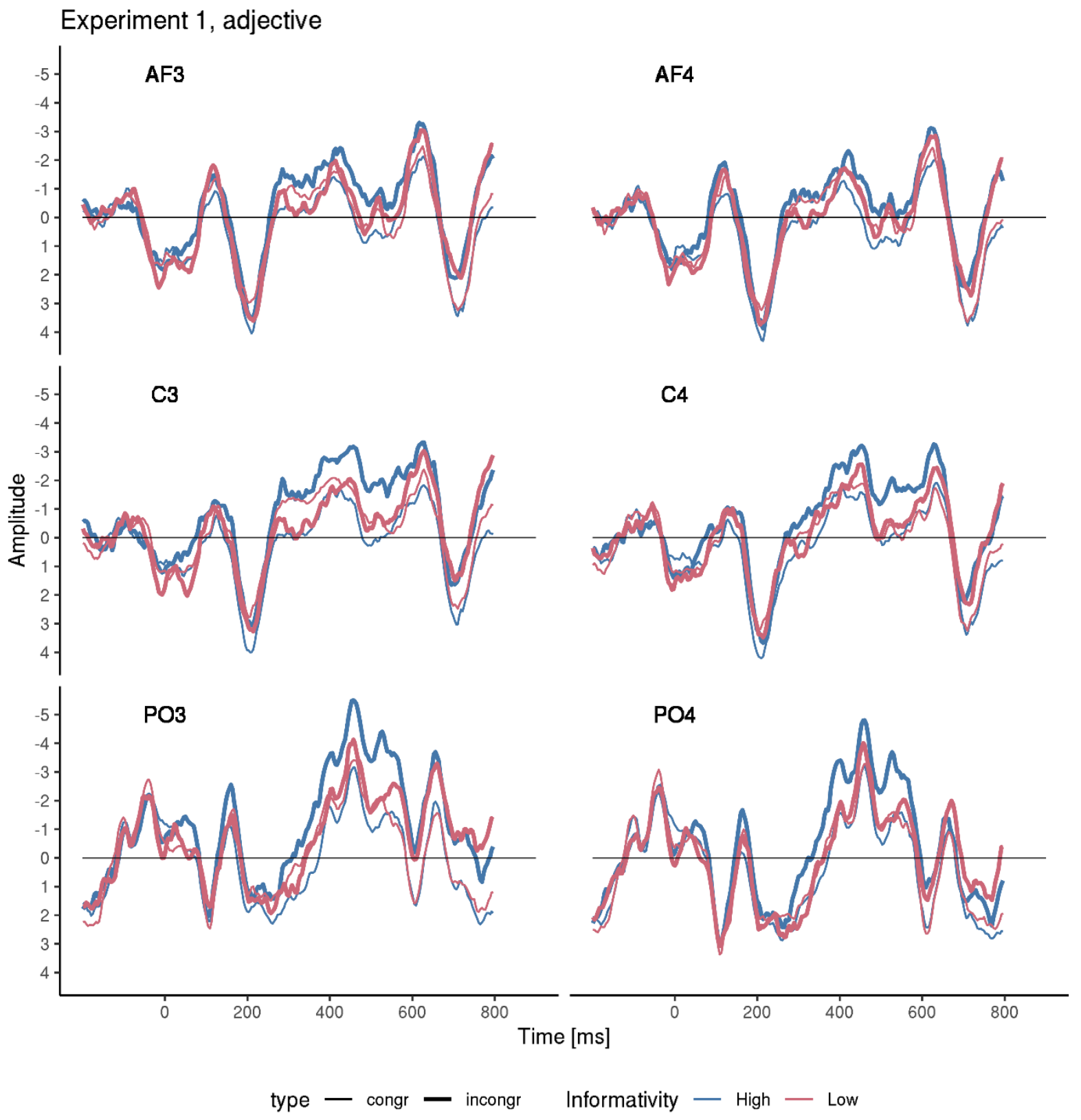

Figure S1. ERPs time-locked to more informative (blue lines) and less informative (red lines) adjectives occurring in congruent (thin lines) and incongruent (bold lines) items in Experiment 1. 


\section{ERPs to nouns in congruent and incongruent items in Experiment 1}

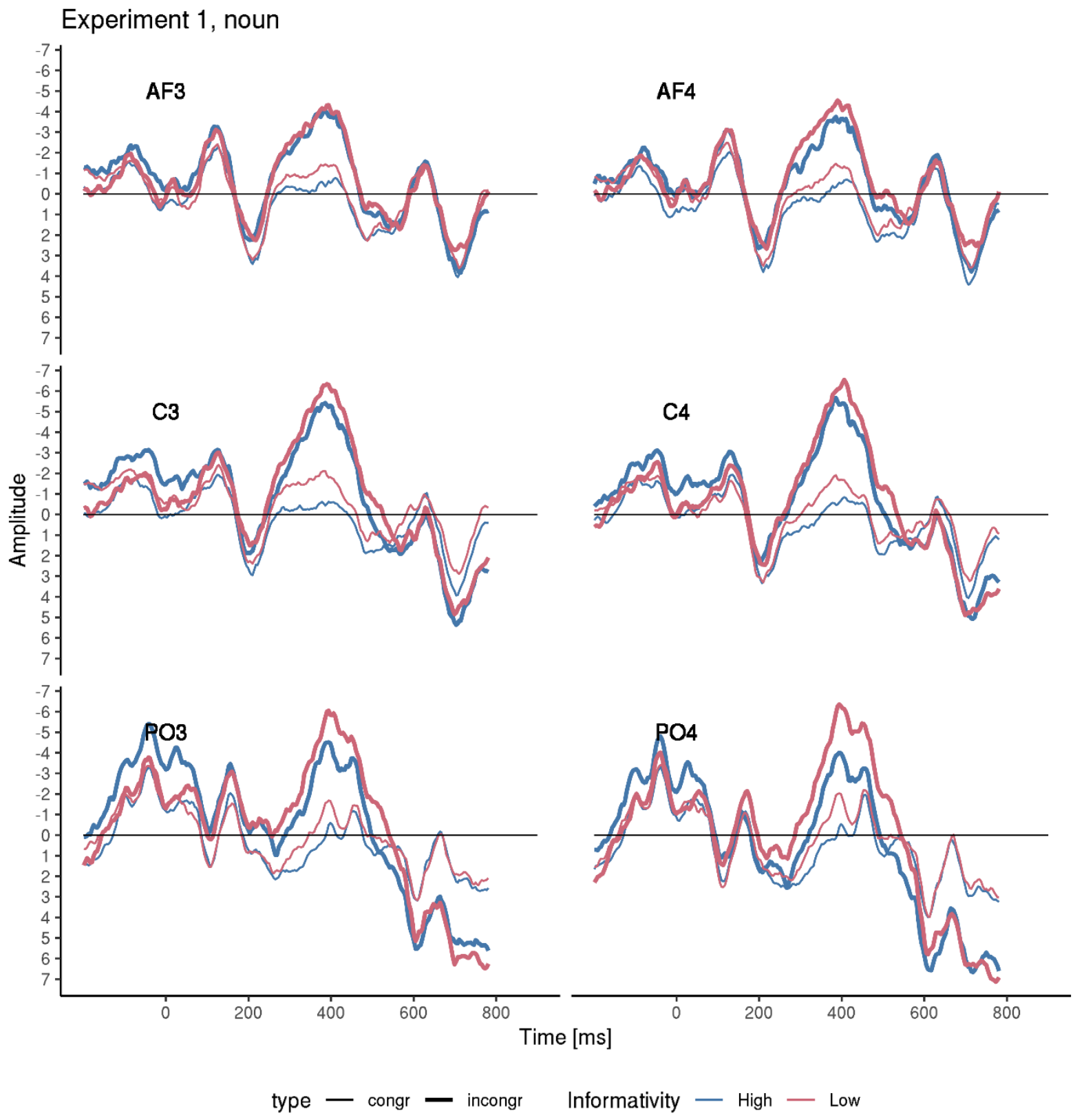

Figure S2. ERPs time-locked to more informative (blue lines) and less informative (red lines) nouns occurring in congruent (thin lines) and incongruent (bold lines) items in Experiment 1 . 


\section{ERPs to adjectives in congruent and incongruent items in Experiment 2}

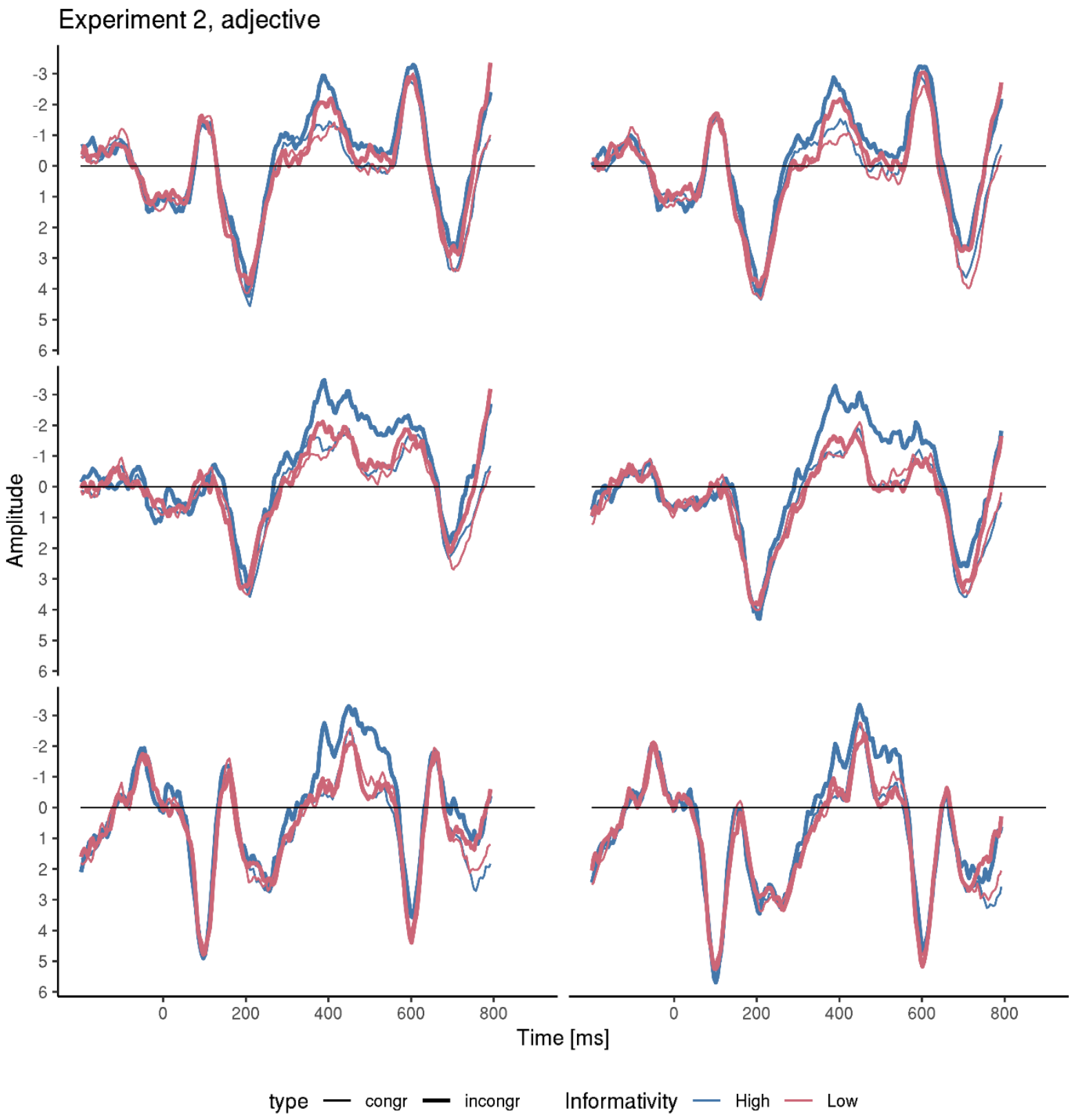

Figure S3. ERPs time-locked to more informative (blue lines) and less informative (red lines) adjectives occurring in congruent (thin lines) and incongruent (bold lines) items in Experiment 2. 


\section{ERPs to nouns in congruent and incongruent items in Experiment 2}

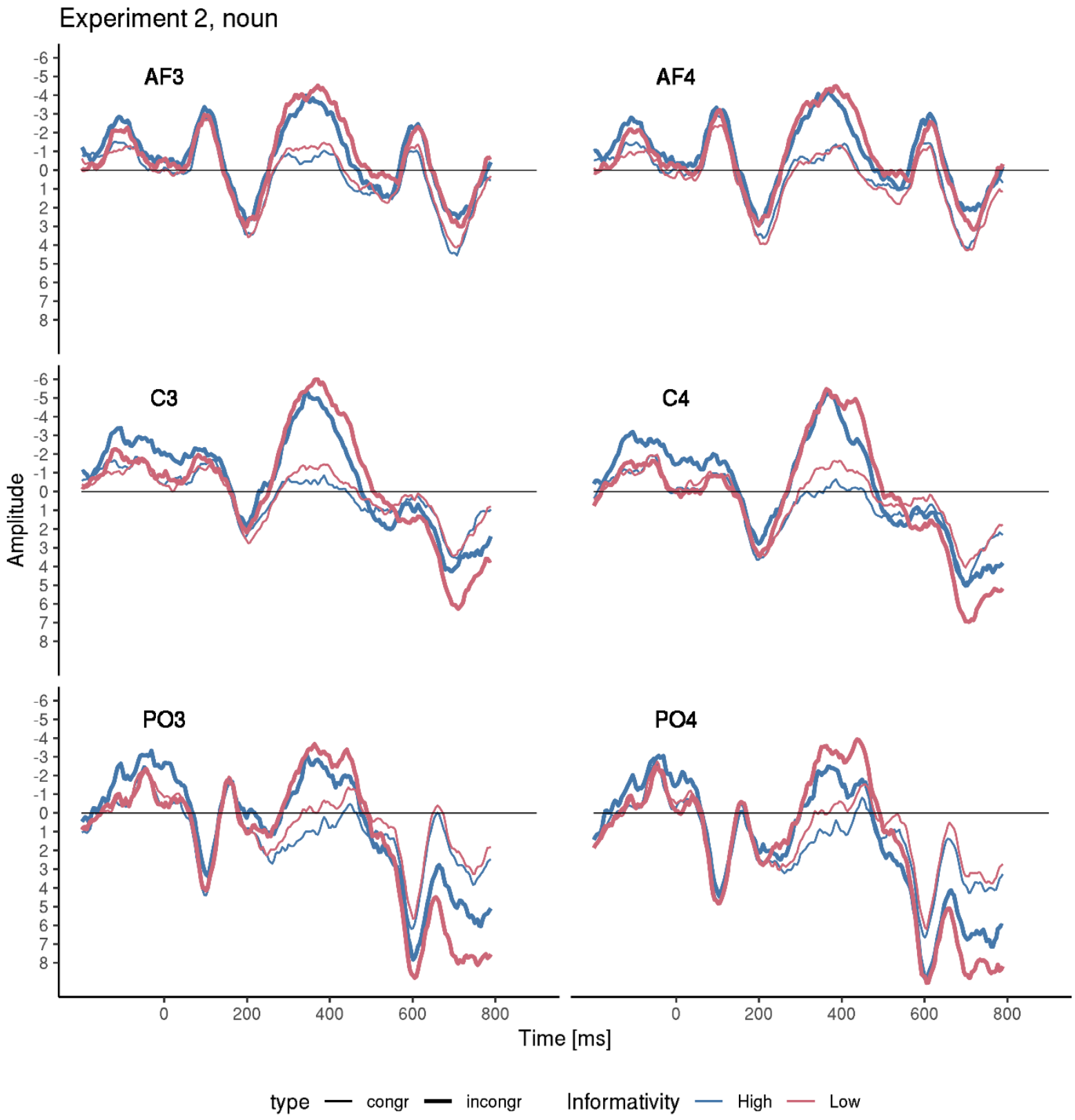

Figure S4. ERPs time-locked to more informative (blue lines) and less informative (red lines) nouns occurring in congruent (thin lines) and incongruent (bold lines) items in Experiment 2 . 


\section{Post-hoc tests addressing potential confounds related to the type of informativity manipulation}

In these post-hoc analyses we wanted to address the question of whether the effect of Informativity changes depending on the way Informativity was manipulated. To this aim, we introduced a new factorial predictor Manipulation Type (2 levels: number feature, grammatical case). In statistical terms, the question was whether Informativity is modified by a significant interaction of Informativity and Manipulation Type. We addressed this question by comparing models with and without the interaction term.

The baseline model contained all predictors present in the main factorial analyses using the Informativity predictor. In addition, the baseline model included a main effect of Manipulation Type, and two by-Subject random slopes: a random slope for Manipulation Type, and a random slope for the interaction of Manipulation Type and Informativity.

The tested model differed from the baseline model only by the addition of the interaction of Informativity and Manipulation Type as a fixed effect. The baseline and the tested model were compared using log-likelihood ratio test. Table $\mathrm{S} 1$ gives the results of the model comparisons for all factorial analyses conducted in Experiment 1 and 2.

\section{Table S1}

Results of model comparisons between a model not including and including the interaction between Informativity and Manipulation Type.

\begin{tabular}{|c|c|c|c|c|}
\hline \multirow[b]{2}{*}{ Analysis } & \multicolumn{2}{|c|}{ Experiment 1} & \multicolumn{2}{|c|}{ Experiment 2} \\
\hline & $\chi^{2}(1)$ & $\mathrm{p}$ & $\chi^{2}(1)$ & $\mathrm{p}$ \\
\hline congruent; adjective & 0.51 & 0.476 & 0.04 & 0.839 \\
\hline congruent; noun & 0.63 & 0.426 & 0.02 & 0.902 \\
\hline incongruent; adjective & 0.53 & 0.467 & 1.42 & 0.233 \\
\hline incongruent; noun & 3.62 & 0.057 & 0.03 & 0.854 \\
\hline
\end{tabular}

As can be seen, in none of the models Manipulation Type significantly improved the fit of the baseline model. 
The mechanisms of prediction updating

\section{Models testing if verbal fluency modulates the magnitude of the effect of informativity on ERP indices of prediction updating}

Table S2

Estimates of fixed and random effects in the LME models using factorial manipulation of adjectival informativity and including predictors related to verbal fluency in Experiment 2.

\begin{tabular}{|c|c|c|c|c|c|c|c|c|c|c|}
\hline \multirow[b]{2}{*}{ Effect } & \multicolumn{5}{|c|}{ Adjectives } & \multicolumn{5}{|c|}{ Nouns } \\
\hline & Estimate & SE & $\mathrm{t}$ & $\begin{array}{l}\text { by- } \\
\text { Item } \\
\text { SD }\end{array}$ & $\begin{array}{c}\text { by- } \\
\text { Subject } \\
\text { SD }\end{array}$ & Estimate & SE & $\mathrm{t}$ & $\begin{array}{l}\text { by- } \\
\text { Item } \\
\text { SD }\end{array}$ & $\begin{array}{c}\text { by- } \\
\text { Subject } \\
\text { SD }\end{array}$ \\
\hline & \multicolumn{10}{|c|}{ Congruent items } \\
\hline Intercept & 0.69 & 0.22 & 3.08 & 0.76 & 1.19 & -2.52 & 0.32 & -7.82 & 1.56 & 1.63 \\
\hline TrialN & 0 & 0.03 & 0.09 & 0.04 & 0.09 & 0.1 & 0.04 & 2.63 & 0.1 & 0.07 \\
\hline Baseline & 6.91 & 0.17 & 41.24 & & 0.87 & 5.76 & 0.29 & 19.68 & 0.88 & 1.59 \\
\hline Informativity & -0.01 & 0.19 & -0.03 & & & 1.19 & 0.3 & 4.01 & 1.26 & \\
\hline Letter Fluency & -0.03 & 0.05 & -0.59 & & & 0.02 & 0.07 & 0.35 & & \\
\hline $\begin{array}{l}\text { Category } \\
\text { Fluency }\end{array}$ & 0.02 & 0.04 & 0.5 & & 0.07 & -0.02 & 0.05 & -0.44 & & 0.06 \\
\hline $\begin{array}{l}\text { Letter Fluency: } \\
\text { Informativity }\end{array}$ & -0.04 & 0.04 & -1 & & & -0.06 & 0.06 & -0.99 & & 0.11 \\
\hline \multirow[t]{2}{*}{$\begin{array}{l}\text { Category } \\
\text { Fluency: } \\
\text { Informativity }\end{array}$} & 0.03 & 0.03 & 0.78 & 0.12 & 0.03 & -0.03 & 0.04 & -0.78 & 0.05 & \\
\hline & \multicolumn{10}{|c|}{ Incongruent items } \\
\hline Intercept & -3.65 & 0.22 & -16.72 & 0.88 & & -5.8 & 0.46 & -12.72 & 1.51 & 2.13 \\
\hline TrialN & -0.01 & 0.03 & -0.35 & 0.06 & & 0.05 & 0.05 & 1.01 & 0.1 & 0.16 \\
\hline Baseline & 7.3 & 0.24 & 30.03 & 0.66 & 1.08 & 5.64 & 0.33 & 17.17 & 1.1 & 1.36 \\
\hline Informativity & -1.05 & 0.27 & -3.9 & & & 1.3 & 0.47 & 2.8 & & 1.55 \\
\hline Letter Fluency & 0 & 0.06 & -0.09 & & 0.24 & 0.06 & 0.1 & 0.63 & & 0.16 \\
\hline $\begin{array}{l}\text { Category } \\
\text { Fluency }\end{array}$ & -0.05 & 0.03 & -2.03 & 0.03 & & -0.03 & 0.07 & -0.43 & & 0.14 \\
\hline $\begin{array}{l}\text { Letter Fluency: } \\
\text { Informativity }\end{array}$ & 0 & 0.07 & 0.05 & 0.19 & & 0.08 & 0.11 & 0.69 & & 0.21 \\
\hline $\begin{array}{l}\text { Category } \\
\text { Fluency: } \\
\text { Informativity }\end{array}$ & 0.03 & 0.05 & 0.61 & 0.13 & & 0.1 & 0.08 & 1.28 & & 0.08 \\
\hline
\end{tabular}

